\title{
A Brownian Motion Version of the Directed Polymer Problem
}

\author{
Joseph G. Conlon' ${ }^{1}$ and Peder A. Olsen ${ }^{1}$
}

Received May 22, 1995

Consider a Brownian particle in three dimensions in a random environment. The environment is determined by a potential random in space and time. It is shown that at small noise the large-time behavior of the particle is diffusive. The diffusion constant depends on the environment. This work generalizes previous results for random walk in a random environment. In these results the diffusion constant does not depend on the environment.

KEY WORDS: Directed polymer; Brownian motion; transfer matrix; spectral gap.

\section{INTRODUCTION}

In this paper we consider a Brownian motion version of the directed polymer problem in a random environment which was considered by Imbrie and Spencer ${ }^{(3)}$ and Bolthausen. ${ }^{(1)}$ The main result of the ImbrieSpencer work is that for a weak random environment in dimension $d \geqslant 3$ the large-time behavior of the polymer problem is given by Brownian motion. Our goal in this paper is to set up a general strategy for proving results of the Imbrie-Spencer type. It will be clear from this that in general one should expect the diffusion constant of the Brownian motion to be renormalized by the average effect of the environment. This should be contrasted with the situation studied in refs. 1 and 3, where there is no renormalization of the diffusion constant. We shall see how a special choice of environment as in refs. 1 and 3 gives rise to no renormalization of the diffusion constant.

${ }^{1}$ Department of Mathematics, University of Michigan, Ann Arbor, Michigan 48109; e-mail: conlon@math.lsa.umich.edu. 
We shall first describe our strategy for the situation when the environment is a Gaussian random field. The remainder of the paper will then be devoted to rigorously implementing this strategy in the simpler situation when the environment consists of variables independent in space and time.

Consider a Gaussian random field $V: \mathbb{R}^{+} \times \mathbb{R}^{d} \rightarrow \mathbb{R}$ with covariance given by

$$
\left\langle V(t, x) V\left(t^{\prime}, x^{\prime}\right)\right\rangle=\varphi\left(x-x^{\prime}\right) \psi\left(t-t^{\prime}\right), \quad x, x^{\prime} \in \mathbb{R}^{d}, \quad t^{\prime} \in \mathbb{P}^{+}
$$

Here the positive-definite functions $\varphi: \mathbb{R}^{d} \rightarrow \mathbb{R}, \psi: \mathbb{R} \rightarrow \mathbb{R}$ are assumed to be shortrange. Hence the varibles $V(t, x), V\left(t^{\prime}, x^{\prime}\right)$ are approximately independent if $\left|t-t^{\prime}\right|$ or $\left|x-x^{\prime}\right|$ is larger than some fixed length scale.

Next let $d W_{x, l}$ be Wiener measure on Brownian paths $X(s), 0 \leqslant s \leqslant t$, in $\mathbb{R}^{d}$ with $X(0)=x$. We define a new probability measure on the paths $X(s), 0 \leqslant s \leqslant t$, by

$$
d W_{x, t, V}=\exp \left[\int_{0}^{t} \varepsilon V(s, X(s)) d s\right] d W_{x, t} / \text { normalization }
$$

where $\varepsilon$ is a parameter which we will choose to be small. We wish to compare the random measure $d W_{x, f, V}$ to the Wiener measure $d W_{x, r}$. To do this recall that for any continuous function $f: \mathbb{R}^{d} \rightarrow \mathbb{R}$ which grows at most exponentially at infinity one has

$$
E_{x}\left[f\left(\frac{X(t)-X(0)}{\sqrt{t}}\right)\right]=E\left[f\left(Y_{1}\right)\right], \quad t>0
$$

where $E_{x}$ denotes expectation with respect to the Wiener measure $d W_{x, t}$ and $Y_{1}$ is the standard normal random variable. The basic question addressed in this paper is to find criteria on $V$ such that

$$
\lim _{t \rightarrow \infty} E_{x}^{V}\left[f\left(\frac{X(t)-X(0)}{\sqrt{t}}\right)\right]=E\left[f\left(Y_{\sigma}\right)\right]
$$

with probability 1 in $V$. Here $E_{x}^{V}$ denotes expectation with respect to the random measure $d W_{x, t, V}$ and $Y_{\sigma}$ is a normal random variable with mean zero and variance $\sigma^{2}$.

The statement (1.3) is reminiscent of the strong law of large numbers. To motivate our strategy for proving (1.3) we recall the proof of the strong law. Suppose that $S_{n}$ is the number of heads in $n$ tosses of a fair coin. The strong law says that

$$
\lim _{n \rightarrow \infty} \frac{S_{n}}{n}=\frac{1}{2} \quad \text { with probability } 1
$$


The number $1 / 2$ is obtained by

$$
\frac{1}{5}=\lim _{n \rightarrow \infty} E\left[S_{n} / n\right]
$$

The probability-1 convergence is obtained by estimating fluctuation as in

$$
E\left[\left|\frac{S_{n}}{n}-\frac{1}{2}\right|^{4}\right] \leqslant \frac{C}{n^{2}}
$$

where $C$ is a universal constant.

If we were to follow the proof of the strong law of large numbers exactly, then our first goal would be to identify the RHS of $(1.3)$ as the limiting expectation of $f((X(t)-X(0)) / \sqrt{t})$ with respect to the measure $E_{V}\left[d W_{x, r, V}\right]$ as $t \rightarrow \infty$. Here $E_{V}$ denotes expectation with respect to $V$. The problem with this is that $E_{v}\left[d W_{x, t, V}\right]$ is a complicated measure since the normalization factor in (1.2) depends on $V$. We try to circumvent this difficulty by replacing $E_{V}\left[d W_{x, 1, v}\right]$ by a simpler measure which we denote by $d W_{x, t, A}$. To define $d W_{x, t, A}$ we first define the partition function $Z_{x, V}(t)$ by

$$
Z_{x, y}(t)=\frac{E_{x}\left\{\exp \left[\int_{0}^{r} \varepsilon V(s, X(s)) d s\right]\right\}}{E_{V} E_{x}\left\{\exp \left[\int_{0}^{t} \varepsilon V(s, X(s)) d s\right]\right\}}
$$

Observe that $E_{v}\left[Z_{x, V}(t)\right]=1$. The measure $d W_{x, t, A}$ is then defined by

$$
d W_{x, t, A}=E_{V}\left[Z_{x, V}(t) d W_{x, t . v}\right]
$$

It is clear that $d W_{x, t, A}$ is a probability measure explicitly given by

$$
d W_{x, t, A}=E_{V},\left\{\exp \left[\int_{0}^{t} \varepsilon V(s, X(s)) d s\right]\right\} d W_{x, t} / \text { normalization }
$$

In the case where $V$ is a Gaussian random field with convariance given by (1.1) one can evaluate the expectation with respect to $V$ in (1.7) to obtain

$$
\begin{aligned}
d W_{x, t, A}= & \exp \left[\frac{\varepsilon^{2}}{2} \int_{0}^{t} \int_{0}^{t} \varphi\left(X(s)-X\left(s^{\prime}\right)\right) \psi\left(s-s^{\prime}\right) d s d s^{\prime}\right] d W_{x, \prime} \\
& \times(\text { normalization })^{-1}
\end{aligned}
$$

The measure $d W_{x, r, A}$ is the measure for a self-attracting Brownian motion with short-range interaction. We shall call it the averaged process. The measure $d W_{x, t, A}$ can be analyzed by the methods of classical statistical mechanics. In fact $d W_{x, t, t}$ is rather like the measure for a one-dimensional 
statistical mechanics system with short-range interaction. It can therefore be controlled by perturbation theory for small $\varepsilon$ or by the transfer matrix method for any value of $\varepsilon^{(5)}$ In particular one should expect that, if $E_{x}^{A}$ denotes expectation with respect to $d W_{x, t, A}$, then

$$
\lim _{t \rightarrow \infty} E_{x}^{A}\left[f\left(\frac{X(t)-X(0)}{\sqrt{t}}\right)\right]=E\left[f\left(Y_{\sigma}\right)\right]
$$

for some normal variable $Y_{\sigma}$ with mean zero and variance $\sigma^{2}$. Equation (1.9) identifies what the RHS of (1.3) should be. Observe that we should in general expect $\sigma \neq 1$ except in the special case when the covariance function $\psi$ in (1.1) is the Dirac $\delta$ function. In that case it is easy to see that $d W_{x, t, A}$ is just the Wiener measure $d W_{x, t}$ again. The situation discussed in refs. 1 and 3 is a discrete version of this.

So far in our discussion we have not required the dimension $d$ to be at least 3 or the parameter $\varepsilon$ in the definition (1.2) of $d W_{x, t . V}$ to be small. These enter because we require fluctuations to be small in analogy to the inequality (1.4) in the proof of the strong law. In view of the definition (1.6) it is clear that the fluctuation of $Z_{x, v}(t)$ should be small. Using the fact that $V$ is a Gaussian random field, we can compute

$$
E_{V}\left[Z_{x, V}(t)^{2}\right]=E_{x, x}^{A}\left[\exp \left[\varepsilon^{2} \int_{0}^{t} \int_{0}^{t} \varphi\left(X(s)-Y\left(s^{\prime}\right)\right) \psi\left(s-s^{\prime}\right) d s d s^{\prime}\right]\right]
$$

where the $X(s), Y(s), 0 \leqslant s \leqslant t$, are independent self-attracting Brownian motions with measure (1.8). In the case when $\psi$ is a Dirac $\delta$ function the RHS of (1.10) is just an expectation value for the quantum two-body problem with interaction potential given by $\varphi$. We can also think of it as a Feynman-Kac integral in $2 d$-dimensional space $\left\{(x, y): x, y \in \mathbb{R}^{d}\right\}$ with potential $\varphi(x-y)$. Since $\varphi$ is short range, the potential $\varphi(x-y)$ is effectively supported on the diagonal set $x-y \simeq 0$, that is, on a set of dimension $d$. Observe that the codimension of this set is also $d$ and that Brownian motion is nonrecurrent to a set if and only if the codimension is larger than 2. It follows that for $d \leqslant 2$ Brownian motion is recurrent to the support of the potential function $\varphi(x-y)$. Hence we should expect the RHS of (1.10) to be large when $d \leqslant 2$, but we can expect it to be $O(1)$ if $d \geqslant 3$. Now even if $d \geqslant 3$ the RHS of $(1.10)$ can still be large, simply by choosing $\varepsilon$ large enough. The reason is that the cost in probability of confining Brownian motion $(X(s), Y(s))$ to a region $|x-X(s)|<\delta$, $|x-Y(s)|<\delta, 0 \leqslant s \leqslant t$, looks like $\exp [-\gamma t]$ for some $\gamma>0$. This small 
probability can be more than offset by the growth in the exponential in the expectation value, which is bounded below by

$$
\exp \left[\varepsilon^{2} t \inf \left\{\varphi\left(x^{\prime}-y^{\prime}\right):\left|x-x^{\prime}\right|<\delta,\left|x-y^{\prime}\right|<\delta\right\}\right]
$$

On the other hand, if $\varepsilon$ is small, this will not be the case and so we can reasonably expect the RHS of (1.10) to be $O(1)$.

Now if the RHS of $(1.10)$ is $O(1)$, then $Z_{x, r}(t)$ cannot become too large, but it does not rule out the possibility of $Z_{x, v}(t)$ coming arbitrarily close to zero as $t \rightarrow \infty$. From the definition (1.6) it is clear that the situation where $Z_{x, v}(t)$ can come close to zero is also dangerous. The problem then of proving that inf ${ }_{r>0} Z_{x, v}(t)$ is bounded away from zero with probability 1 in $V$ is a central issue. Once this issue has been resolved the proof of (1.3) follows from (1.9) by estimating mean square fluctuations or higher moments as in the proof of the strong law. We consider the process $X(t)$ with measure $d W_{x, r, V}$ at some large time, say $t^{1 / 3}$. Since $\inf _{s>0} Z_{x, V}(s)$ is bounded away from zero, the density for $X\left(t^{1 / 3}\right)$ is spread out on a length scale $t^{1 / 6}$. Now we think of $X(t)$ as starting at time $t^{1 / 3}$ from a density spread out on a length scale $t^{1 / 6}$. The fluctuation will then be proportional to some power of $t^{-1 / 6}$ and hence goes to zero as $t \rightarrow \infty$.

We shall implement now the strategy described above for field variables $V(t, x)$ which are independent on length scales larger than 1 .

We consider independent Bernoulli random variables $V_{i, n}, i=1,2, \ldots$, $n \in \mathbb{Z}^{d}$. For $n \in \mathbb{Z}^{d}$ let $Q_{n}$ be the unit cube centered at $n$. Our random environment is then a function $V: \mathbb{R}^{+} \times \mathbb{R}^{d} \rightarrow\{-1,1\}$ defined by

$$
V(t, x)=V_{i, n}, \quad i-1<t \leqslant i, \quad x \in Q_{n}
$$

The main theorem we shall prove here is the following:

Theorem 1.1. Let $V$ be given by (1.11) and $f: \mathbb{R}^{d} \rightarrow \mathbb{R}$ be a measurable function satisfying the condition

$$
\int_{\mathbb{R}^{\prime \prime}} e^{-|y|}|f(y)|^{2} d y<\infty
$$

Then if $\varepsilon>0$ is sufficiently small and $d \geqslant 3$, one has that

$$
\begin{gathered}
\lim _{n \rightarrow \infty, n \in \mathbb{Z}, t=2^{n}} \frac{E_{x}\left\{\exp \left[\int_{0}^{t} \varepsilon V(s, X(s)) d s\right] f((X(t)-X(0)) / \sqrt{t})\right\}}{E_{x}\left\{\exp \left[\int_{0}^{t} \varepsilon V(s, X(s)) d s\right]\right\}} \\
=\frac{1}{\left(2 \pi \sigma^{2}\right)^{d / 2}} \int_{\mathbb{R}^{d}} \exp \left(\frac{-|y|^{2}}{2 \sigma^{2}}\right) f(y) d y
\end{gathered}
$$

with probability 1 in $V$. The diffusion constant $\sigma$ depends only on $\varepsilon, d$. 
Remark. In Theorem 1.1 we require the values $t$ to go to infinity exponentially fast in (1.12). This is a technical requirement which we must impose since we confine ourselves in this paper to estimating only mean square fluctuations. Just as in the proof of the strong law of large numbers, one must estimate higher moments to obtain convergence with probability 1 along the entire integer sequence.

A key ingredient in the proof of Theorem 1.1 will be to show that the partition function (1.5) converges as $t \rightarrow \infty$ to a function $Z_{x, V}(\infty)$. This function is trivially nonnegative. We shall need to show that it is strictly positive with probability 1 . In fact we can show that

$$
P\left(Z_{x, r}(\infty)<\delta\right) \leqslant C /|\log \delta|^{\alpha}
$$

where $\delta<1 / 2$ is arbitrarily small and $C, \alpha$ are positive constants independent of $\delta$. It would be interesting to show that the limit in (1.12) holds if one takes the expectation value with respect to $V$ on the LHS. This of course does not follow from Theorem 1.1 since almost everywhere convergence of a sequence does not imply that integrals converge. To prove such a theorem it appears that one would need to sharpen the inequality (1.13) to have $C \delta^{\alpha}$ on the RHS. We are unable to prove this.

Our first task here will be to study the averaged process (1.7). In Section 2 we shall show by using the transfer matrix method that the measure (1.7) is similar in many respects to the Wiener measure. In particular the recurrence properties of the measure (1.7) are the same as for the Wiener measure. However, the mean square deviation of $X(t)$ at large $t$ is different. The constant $\sigma$ in (1.2) is given by

$$
\lim _{t \rightarrow \infty} t^{-1} E_{x}^{A}\left[(X(t)-X(0))^{2}\right]=\sigma^{2}
$$

and $\sigma \neq 1$ in general.

\section{THE AVERAGED PROCESS}

In this section we shall use the transfer matrix method of statistical mechanics $^{(5)}$ to analyze the measure (1.7). First let us consider the normalization factor with $t=N$ an integer

$$
\begin{aligned}
E_{x} E_{V} & \left\{\exp \left[\int_{0}^{N} \varepsilon V(s, X(s)) d s\right]\right\} \\
& =E_{x}\left[\prod_{i=1}^{N} E_{V}\left\{\exp \left[\int_{i-1}^{i} \varepsilon V(s, X(s)) d s\right]\right\}\right]
\end{aligned}
$$


Let us denote by $K(x, y), x, y \in \mathbb{R}^{d}$, the kernel defined by

$$
K(x, y)=E_{x, y} E_{V}\left\{\exp \left[\int_{0}^{1} \varepsilon V(s, X(s) d s]\right\} \frac{1}{(2 \pi)^{d / 2}} \exp \left(\frac{-|x-y|^{2}}{2}\right)\right.
$$

where $E_{x, y}$ indicates that the expectation is taken with respect to Brownian motion $X(t)$ conditioned on $X(0)=x, X(1)=y$. Thus we have

$$
\begin{aligned}
& E_{x} E_{V}\left\{\exp \left[\int_{0}^{N} \varepsilon V(s, X(s)) d s\right]\right\} \\
& \quad=\int_{\mathbb{R}^{d}} \cdots \int_{\mathbb{R}^{d}} d y_{1} d y_{2} \cdots d y_{N} K\left(x, y_{1}\right) K\left(y_{1}, y_{2}\right) \cdots K\left(y_{N-1}, y_{N}\right)
\end{aligned}
$$

Next observe that $K$ is translation invariant, whence

$$
K(x+n, y+n)=K(x, y), \quad n \in \mathbb{Z}^{d}
$$

We may write the integral in (2.2) as integrals over the unit cube $Q_{0}$ in $\mathbb{R}^{d}$ centered at the origin together with sums over $n \in \mathbb{Z}^{d}$. Thus

$$
\begin{aligned}
\int_{\mathbb{R}^{d}} \cdots & \int_{\mathbb{R}^{d}} d y_{1} d y_{2} \cdots d y_{N} K\left(x, y_{1}\right) K\left(y_{1}, y_{2}\right) \cdots K\left(y_{N-1}, y_{N}\right) \\
= & \sum_{m_{1} \in \mathbb{Z}^{d}} \cdots \sum_{n_{N} \in \mathbb{Z}^{d}} \int_{Q_{0}} \cdots \int_{Q_{0}} d y_{1} \cdots d y_{N} \\
& \times K\left(x, y_{1}+n_{1}\right) K\left(y_{1}+n_{1}, y_{2}+n_{2}\right) \cdots K\left(y_{N-1}+n_{N-1}, y_{N}+n_{N}\right) \\
= & \sum_{m_{1} \in \mathbb{Z}^{d}} \cdots \sum_{m_{N} \in \mathbb{Z}^{d}} \int_{Q_{0}} \cdots \int_{Q_{0}} d y_{1} \cdots d y_{N} \\
& \times K\left(x, y_{1}+m_{1}\right) K\left(y_{1}, y_{2}+m_{2}\right) \cdots K\left(y_{N-1}, y_{N}+m_{N}\right)
\end{aligned}
$$

where we have used the translation invariance (2.3) in the last identity. If we define now a new kernel $K_{0}$ on $Q_{0} \times Q_{0}$ by

$$
K_{0}(x, y)=\sum_{m \in \mathbb{Z}^{d}} K(x, y+m)
$$

then we have that

$$
\begin{aligned}
E_{x} E_{V} & \left\{\exp \left[\int_{0}^{N} \varepsilon V(s, X(s)) d s\right]\right\} \\
& =\int_{Q_{0}} \cdots \int_{Q_{0}} d y_{1} \cdots d y_{N} K_{0}\left(\bar{x}, y_{1}\right) K_{0}\left(y_{1}, y_{2}\right) \cdots K_{0}\left(y_{N-1}, y_{N}\right)
\end{aligned}
$$


where $\bar{x} \in Q_{0}$ is determined by $\bar{x}-x \in \mathbb{Z}^{d}$. We may write the last expression in operator notation. Let $A_{0}$ be the operator on $L^{2}\left(Q_{0}\right)$ defined by

$$
A_{0} f(x)=\int_{Q_{0}} K_{0}(x, y) f(y) d y
$$

Then we finally have

$$
E_{x} E_{V}\left\{\exp \left[\int_{0}^{N} \varepsilon V(s, X(s)) d s\right]\right\}=\left\langle\delta_{\bar{x}}, A_{0}^{N} \chi_{0}\right\rangle
$$

where $\delta_{\bar{x}}$ is the Dirac $\delta$ function centered at $\bar{x}$, and for $\xi \in \mathbb{C}^{d}, \chi_{\xi}$ is the function $\chi_{\xi}(y)=\exp [i \xi \cdot y], y \in Q_{0}$.

We shall be interested in computing the Fourier transform $\phi_{r}(\zeta)$, $\xi \in \mathbb{R}^{\prime \prime}$, given by

$$
\phi_{r}(\xi)=E_{x}^{A}[\exp [i \xi \cdot(X(t)-X(0))]]
$$

We can obtain an expression for $\phi_{r}(\xi)$ of the form (2.4) by observing that

$$
\begin{aligned}
E_{x} E_{V}[ & \left.\exp \left[\int_{0}^{N} \varepsilon V(s, X(s)) d s\right] \exp [i \xi \cdot(X(N)-X(0))]\right] \\
= & \int_{Q_{v}} \cdots \int_{Q_{v}} d y_{1} \cdots d y_{N} \\
& \quad \times \exp [-i \xi \cdot \bar{x}] K_{\xi}\left(\bar{x}, y_{1}\right) K_{\xi}\left(y_{1}, y_{2}\right) \cdots K_{\bar{\xi}}\left(y_{N-1}, y_{N}\right) \exp \left[i \xi \cdot y_{N}\right]
\end{aligned}
$$

where the kernel $K_{\zeta}$ is given by

$$
K_{\xi}(x, y)=\sum_{m \in \mathbb{Z}^{d}} K(x, y+m) e^{i \xi \cdot m}
$$

Hence if $A_{\xi}$ is the operator on $L^{2}\left(Q_{0}\right)$ given by

$$
A_{\xi} f(x)=\int_{Q_{0}} K_{\xi}(x, y) f(y) d y
$$

we have

$$
\phi_{N}(\xi)=\left\langle\chi_{\bar{\xi}}(\bar{x}) \delta_{\bar{N}}, A_{\bar{\xi}}^{N} \chi_{\bar{\xi}}\right\rangle\left\langle\left\langle\delta_{\overline{.}}, A_{0}^{N} \chi_{0}\right\rangle\right.
$$

In (2.7) we are using the convention

$$
\langle\phi, \psi\rangle=\int_{Q_{0}} \overline{\phi(x)} \psi(x) d x
$$


Our first task is to investigate the structure of the operator $A_{\xi}$. Toward that goal we prove some properties of the kernel $K_{\xi}$ :

Lemma 2.1. The kernel $K_{\xi}$ has the following properties:

(a) $K_{\xi}(x, y)$ as a function of $(x, y, \xi), x, y \in \mathbb{R}^{d}, \xi \in \mathbb{C}^{d}$, is continuous in $x, y$ and analytic in $\xi$.

(b) $K_{\xi}, \xi \in \mathbb{C}^{d}$, is periodic, $K_{\xi+2 \pi n}=K_{\xi}, n \in \mathbb{Z}^{d}$, the $d$-dimensional integer lattice.

(c) For $x, y, \xi \in \mathbb{R}^{d}$ there is the identity

$$
K_{\xi}(x, y)=\overline{K_{\xi}(y, x)}
$$

(d) $K_{0}(x, y)>0, \quad x, y \in \mathbb{R}^{d}$.

Proof. (a) The kernel $K(x, y)$ given by $(2.1)$ is continuous in $x, y$ and satisfies an inequality

$$
0<K(x, y) \leqslant C \exp \left[-|x-y|^{2} / 2\right], \quad x, y \in \mathbb{R}^{d}
$$

The continuity follows by writing the conditional expectation $E_{x_{,},}$as a Brownian bridge expectation value. Continuity in $x, y$ is then a consequence of the dominated convergence theorem. The continuity and analyticity of $K_{\xi}(x, y)$ follow from the continuity of $K(x, y)$ and the bound (2.8).

(b) This is immediate from (2.6).

(c) Observe that $K(x, y)=K(y, x), x, y \in \mathbb{R}^{d}$. This follows from the time-reversal invariance of conditional Brownian motion. To get (c) we use (2.3) to obtain

$$
\begin{aligned}
\overline{K_{\xi}(x, y)} & =\sum_{m \in \mathbb{Z}^{\prime}} K(x, y+m) e^{-i \xi \cdot m} \\
& =\sum_{m \in \mathbb{Z}^{\prime}} K(x, y-m) e^{i \xi \cdot m}=\sum_{m \in \mathbb{Z}^{d}} K(x+m, y) e^{i \xi \cdot m} \\
& =\sum_{m \in \mathbb{Z}^{\prime}} K(y, x+m) e^{i \xi \cdot m}=K_{\xi}(y, x)
\end{aligned}
$$

(d) This is immediate from (2.1).

Proposition 2.1. The operator $A_{\xi}$ on $L^{2}\left(Q_{0}\right)$ has the following properties:

(a) $A_{\xi}$ is a compact operator for $\xi \in \mathbb{C}^{d}$.

(b) $A_{\xi}$ is self-adjoint for $\xi \in \mathbb{R}^{d}$. 
(c) The function $\xi \rightarrow A_{\xi}$ is strongly continuous for $\xi \in \mathbb{C}^{d}$.

(d) $A_{0}$ has an eigenvector $u_{0}$ with eigenvalue $\left\|A_{0}\right\|$. The function $u_{0}(x), x \in Q_{0}$, is continuous and strictly positive, i.e., $u_{0}(x)>0$, $x \in Q_{0}$. There exists $\gamma<1$ such that $|\lambda| \leqslant \gamma\left\|A_{0}\right\|$ for all other eigenvalues $\lambda$ of $A_{0}$.

Proof. (a) This follows from (a) of Lemma 2.1 and the Ascoli-Arzela theorem.

(b) This follows from (c) of Lemma 2.1.

(c) This follows from (2.6) and (2.8).

(d) This follows from (d) of Lemma 2.1 and the Krein-Rutman theorem. ${ }^{(4)}$

Let $\rho_{r}(y), y \in \mathbb{R}^{d}$, be the Fourier inverse of $\phi_{r}(\xi)$. We shall use Lemma 2.1 and Proposition 2.1 to estimate $\rho_{t}$ for large $t$.

Proposition 2.2. There exist constants $C_{1}, C_{2} \geqslant 0$ such that

$$
0 \leqslant \rho_{i}(y) \leqslant \frac{C_{1}}{t^{d / 2}} \exp \left(\frac{-c_{2}|y|}{t^{1 / 2}}\right), \quad t>0
$$

Proof. We first estimate the denominator in (2.7). We have

$$
\left\langle\delta_{\bar{x}}, A_{0}^{N} \chi_{0}\right\rangle=\left\langle A_{0} \delta_{\bar{x}}, A_{0}^{N-1} \chi_{0}\right\rangle
$$

where $A_{0} \delta_{\bar{x}} \in L^{2}\left(Q_{0}\right)$. Next observe that

$$
\begin{aligned}
A_{0}^{N-1} \chi_{0} & =A_{0}^{N-1}\left[\left\langle u_{0}, \chi_{0}\right\rangle u_{0}+P \chi_{0}\right] \\
& =\left\langle u, \chi_{0}\right\rangle\left\|A_{0}\right\|^{N-1} u_{0}+A_{0}^{N-1} P \chi_{0}
\end{aligned}
$$

where $P$ is projection orthogonal to $u_{0}$. In view of Proposition 2.1(d) we have that

$$
\left\|A_{0}^{N-1} \chi_{0}-\left\langle u, \chi_{0}\right\rangle\right\| A_{0}\left\|^{N-1} u_{0}\right\| \leqslant \gamma^{N-1}\left\|A_{0}\right\|^{N-1}\left\|\chi_{0}\right\|
$$

Hence we have the inequality

$$
\begin{aligned}
\left\|A_{0}\right\|^{N-1}\left[\left\langle A_{0} \delta_{\bar{x}}, u_{0}\right\rangle\left\langle u_{0}, \chi_{0}\right\rangle-\gamma^{N-1}\left\|A_{0} \delta_{\bar{x}}\right\| \cdot\left\|\chi_{0}\right\|\right] \\
\quad \leqslant\left\langle A_{0} \delta_{\bar{x}}, A_{0}^{N-1} \chi_{0}\right\rangle \\
\quad \leqslant\left\|A_{0}\right\|^{N-1}\left[\left\langle A_{0} \delta_{\bar{x}}, u_{0}\right\rangle\left\langle u_{0}, \chi_{0}\right\rangle+\gamma^{N-1}\left\|A_{0} \delta_{\bar{x}}\right\| \cdot\left\|\chi_{0}\right\|\right]
\end{aligned}
$$

Since $\left\langle u_{0}, \chi_{0}\right\rangle>0$ and $\left\langle A_{0} \delta_{\bar{x}}, u_{0}\right\rangle>0$, by Proposition 2.1 , it follows that the denominator of (2.7) behaves like $\left\|A_{0}\right\|^{N-1}$ for large $N$. 
Next we consider the numerator of (2.7). We have

$$
\begin{aligned}
\sum_{n \in \mathbb{Z}^{\prime}} & \left\langle\chi_{\xi+2 \pi n}(\bar{x}) \delta_{\bar{x}}, A_{\bar{\xi}+2 \pi n}^{N} \chi_{\xi+2 \pi n}\right\rangle \exp [-i(\xi+2 \pi n) \cdot y] \\
& =\left\langle\chi_{\xi}(\bar{x}) \delta_{\bar{x}}, A_{\bar{\xi}}^{N}\left\{\chi_{\xi} \sum_{n \in \mathbb{Z}^{\prime}} \chi_{2 \pi n} \exp [-2 \pi n i \cdot(\bar{x}+y)]\right\}\right\rangle \exp [-i \xi \cdot y] \\
& =\left\langle\chi_{\xi}(\bar{x}) \delta_{x}, A_{\xi}^{N}\left[\chi_{\bar{\xi}}(\overline{x+y}) \delta_{\overline{x+y}}\right]\right\rangle \exp [-i \xi \cdot y]
\end{aligned}
$$

In the final step above we used the Poisson summation formula. Hence we obtain the following formula for $p_{N}(y)$ :

$$
\begin{aligned}
\rho_{N}(y)= & \left\{\int_{J} d \xi\left\langle\chi_{\bar{\xi}}(\bar{x}) \delta_{\bar{x}}, A_{\bar{\xi}}^{N}\left[\chi_{\xi}(\overline{x+y}) \delta_{\overline{x+y}}\right]\right\rangle \exp [-i \xi \cdot y]\right\} \\
& \times\left[(2 \pi)^{d}\left\langle\delta_{\bar{x}}, A_{0}^{N} \chi_{0}\right\rangle\right]^{-1}
\end{aligned}
$$

where $J$ is the cube $[-\pi, \pi]^{d}$.

To estimate $(2.11)$ we shall prove that there exists a constant $c>0$ such that

$$
\left\|A_{\xi}\right\| \leqslant\left\|A_{0}\right\|\left(1-c|\xi|^{2}\right), \quad \xi \in J
$$

First we consider the case when $|\xi|$ is small. Let us write $\xi=t n$, where $n$ is a unit vector and $t$ a scalar. Then we have

$$
A_{5}=A_{0}+t A_{1}+t^{2} A_{2}+O\left(t^{3}\right)
$$

Let $P$ be a coordinate plane through the origin in $\mathbb{R}^{d}$ and let $\pi_{p}$ denote reflection in this plane. It is easy to see that the kernel $K_{0}$ of $A_{0}$ has the property

$$
K_{0}\left(\pi_{P}(x), \pi_{P}(y)\right)=K_{0}(x, y), \quad x, y \in Q_{0}
$$

It follows by the uniqueness of the eigenvector $u_{0}$ that $u_{0}\left(\pi_{P}(x)\right)=u_{0}(x)$, $x \in \mathbb{Q}_{0}$. Now if the unit vector is along a coordinate axis, the kernel $K_{1}$ of $A_{1}$ has the property

$$
K_{1}\left(\pi_{P}(x), \pi_{P}(y)\right)=-K_{1}(x, y), \quad x, y \in Q_{0}
$$

We concli that for all directions $n$ one has

$$
\left\langle u_{0}, A_{1} u_{0}\right\rangle=0
$$

whence the vector $A_{1} u_{0}$ is orthogonal to $u_{0}$. 
In view of the analyticity of $A_{\xi}$ in $\xi$ there is an eigenvector $u_{\xi}$ of $A_{\xi}$, analytic in $\xi$ in a neighborhood of $\xi=0$. We can seek this eigenvector by expansion

$$
u_{\xi}=a(\xi) u_{0}+v(\xi)
$$

where $a(0)=1, v(0)=0$, and the vector $v(\xi)$ is orthogonal to $u_{0}$. We can expand $a(\xi), v(\xi)$ in powers of $t$,

$$
\begin{aligned}
& a(\xi)=1+a_{1} t+a_{2} t^{2}+\cdots \\
& v(\xi)=v_{1} t+v_{2} t^{2}+\cdots
\end{aligned}
$$

Evidently all the vectors $v_{1}, v_{2}, \ldots$ are orthogonal to $u_{0}$. For $\xi$ real the eigenvalue of $u_{\xi}$ is given by $\left\|A_{\xi}\right\|$, which also has an expansion

$$
\left\|A_{\vdots}\right\|=\left\|A_{0}\right\|+\lambda_{1} t+\lambda_{2} t^{2}+\cdots
$$

We can compute the coefficients in the expansion by using the equation $A_{\xi} u_{\xi}=\left\|A_{\xi}\right\| u_{\xi}$ and identifying coefficients of powers of $t$. Thus

$$
\begin{aligned}
& {\left[A_{0}+t A_{1}+t^{2} A_{2}+\cdots\right]\left\{\left(1+a_{1} t+a_{2} t^{2}+\cdots\right) u_{0}+v_{1} t+v_{2} t^{2}+\cdots\right\}} \\
& \quad=\left[\left\|A_{0}\right\|+\lambda_{1} t+\lambda_{2} t^{2}+\cdots\right]\left\{\left(1+a_{1} t+a_{2} t^{2}+\cdots\right) u_{0}+v_{1} t+v_{2} t^{2}+\cdots\right\}
\end{aligned}
$$

Equating the coefficients of $t$ on both sides of the equation, we have

$$
a_{1} A_{0} u_{0}+A_{1} u_{0}+A_{0} v_{1}=a_{1}\left\|A_{0}\right\| u_{0}+\lambda_{1} u_{0}+\left\|A_{0}\right\| v_{1}
$$

which is the same as

$$
A_{1} u_{0}+A_{0} v_{1}=\lambda_{1} u_{0}+\left\|A_{0}\right\| v_{1}
$$

If we take the scalar product of $u_{0}$ with this last equation and use the fact that both $v_{1}$ and $A_{1} u_{0}$ are orthogonal to $u_{0}$ we conclude that $\lambda_{1}=0$. Hence $v_{1}$ is given by the formula

$$
v_{1}=\left(\left\|A_{0}\right\|-A_{0}\right)^{-1} A_{1} u_{0}
$$

Next we equate the coefficients of $t^{2}$ and put $\lambda_{1}$ equal to zero. Thus we have

$$
\begin{gathered}
a_{2} A_{0} u_{0}+a_{1} A_{1} u_{0}+A_{2} u_{0}+A_{0} v_{2}+A_{1} v_{1} \\
=a_{2}\left\|A_{0}\right\| u_{0}+\lambda_{2} u_{0}+\left\|A_{0}\right\| v_{2}
\end{gathered}
$$


which is the same as

$$
a_{1} A_{1} u_{0}+A_{2} u_{0}+A_{0} v_{2}+A_{1} v_{1}=\lambda_{2} u_{0}+\left\|A_{0}\right\| v_{2}
$$

Taking the scalar product of the last equation with $u_{0}$ and using the fact that $v_{2}$ and $A_{1} u_{0}$ are orthogonal to $u_{0}$, we conclude that

$$
\lambda_{2}=\left\langle u_{0}, A_{2} u_{0}\right\rangle+\left\langle u_{0}, A_{1} v_{1}\right\rangle
$$

Since $A_{\xi}$ is self-adjoint for $\xi \in \mathbb{R}^{d}$, it follows that $A_{1}$ is self-adjoint. Thus we have

$$
\lambda_{2}=\left\langle u_{0}, A_{2} u_{0}\right\rangle+\left\langle A_{1} u_{0},\left(\left\|A_{0}\right\|-A_{0}\right)^{-1} A_{1} u_{0}\right\rangle
$$

It is clear that the first term on the right in the last equation is less than or equal to zero. Since $\left(\left\|A_{0}\right\|-A_{0}\right)^{-1}$ is nonnegative definite, the second term in the last equation is greater than or equal to zero. Hence it is not obvious that $\lambda_{2}$ is negative. We shall prove in the Appendix that $\lambda_{2}<0$. Thus for $|\xi|$ small we have

$$
\left\|A_{\xi}\right\|=\left\|A_{0}\right\|+Q(\xi, \xi)+O\left(|\xi|^{3}\right)
$$

where $Q$ is a negative-definite quadratic form. Thus there is a constant $c>0$ such that $Q(\xi, \xi) \leqslant-c\left\|A_{0}\right\| \cdot|\xi|^{2}$. We have proved therefore the inequality (2.12) in some neighborhood of $\xi=0$.

To complete the proof of (2.12) observe that for any $\delta>0$ there is a constant $\varepsilon>0$ such that

$$
\left|K_{\xi}(x, y)\right| \leqslant(1-\varepsilon) K_{0}(x, y), \quad x, y \in Q_{0}, \quad \xi \in J,|\xi|>\delta
$$

Thus $\left\|A_{\xi}\right\| \leqslant(1-\varepsilon)\left\|A_{0}\right\|$ for $\xi \in J,|\xi|>\delta$. The inequality follows by choosing $c$ sufficiently small so that $c\left\|A_{0}\right\| \cdot|\xi|^{2}<\varepsilon, \xi \in J$.

Let us take $t=N$, an integer, in (2.9). Then the inequality (2.12) immediately implies (2.9) with $y=0$. In fact from (2.11) we have

$$
\begin{aligned}
\left|\rho_{N}(y)\right| & \leqslant C \int_{J}\left[1-c|\xi|^{2}\right]^{N} d \xi \\
& \leqslant C \int_{J} \exp \left[-c N|\xi|^{2}\right] d \xi \leqslant C_{1} / N^{d / 2}
\end{aligned}
$$

for some constant $C_{1}$ independent of $N$. Observe that we have used the fact that

$$
\sup _{\xi \in J}\left\|A_{\zeta} \chi_{\xi}(\bar{x}) \delta_{. \bar{x}}\right\|<\infty
$$


In order to prove (2.9) for $|y|>N^{1 / 2}$ we need to deform the integration in (2.11) to an integration involving complex $\xi$. Observe that by Lemma 2.1(b) the integrand in $(2.11)$ is periodic. Thus

$$
\begin{aligned}
\left\langle\chi_{\xi-\pi n}(\bar{x}) \delta_{\bar{x}}, A_{\bar{\xi}-\pi n}^{N}\left[\chi_{\xi-\pi n}(\overline{x+y}) \delta_{\overline{x+y}}\right]\right\rangle \exp [-i(\xi-\pi n) \cdot y] \\
=\left\langle\chi_{\xi+\pi n}(\bar{x}) \delta_{\bar{x}}, A_{\bar{\xi}+\pi n}^{N}\left[\chi_{\bar{\xi}+\pi n}(\overline{x+y}) \delta_{\overline{x+y}}\right]\right\rangle \\
\quad \times \exp [-i(\xi+\pi n) \cdot y], \quad \xi \in \mathbb{C}^{d}, \quad n \in \mathbb{Z}^{d}, \quad x, y \in \mathbb{R}^{d}
\end{aligned}
$$

Hence we may deform the integration $d \xi=d \xi_{1} \cdots d \xi_{d}$ on $[-\pi, \pi]^{d}$ to $d\left(\xi_{1}+i \eta_{1}\right) \cdots d\left(\xi_{d}+i \eta_{d}\right)$, where $\eta_{1}, \ldots, \eta_{d}$ are arbitrary real parameters. We choose the $\eta_{j}, j=1, \ldots, d$, by $\eta_{j}=\operatorname{sgn}\left(y_{j}\right) / N^{1 / 2}$. The inequality (2.9) follows for all $y \in \mathbb{R}^{d}$ provided we can show that for any $\alpha>0$ there exist positive constants $c, d>0$ such that

$$
\left\|A_{\xi+i \eta}\right\| \leqslant\left\|A_{0}\right\|\left(1-c|\xi|^{2}+d|\eta|^{2}\right), \quad \xi \in J, \quad|\eta|<\alpha
$$

Observe that it is again sufficient to prove (2.14) provided $\xi+i \eta$ are in a small neighborhood of the origin. In that case the self-adjoint operator $A_{\xi+i \eta}^{*} A_{\xi+i \eta}$ has a unique eigenvector $u_{\xi+i \eta}$ whose eigenvalue is $\left\|A_{\xi+i \eta}\right\|^{2}$. When $\eta=0$ this eigenvector coincides with the previous eigenvectors $u_{\xi}$. Now it is easy to see that

$$
A_{\xi+i \eta}^{*} A_{\xi+i \eta}=A_{\xi-i \eta} A_{\xi+i \eta}
$$

Hence, as before, $\left\|A_{\xi+i \eta}\right\|^{2}$ is analytic in $\xi, \eta$ close to the origin. We need to show that in a power series expansion in $\xi, \eta$ the linear term in $\eta$ is zero. To do this let us write $\eta=t n$, where $n$ is a unit vector and $t$ real. Then

$$
\begin{aligned}
A_{i \eta} & =A_{0}+t A_{1}+\cdots \\
u_{i \eta \eta} & =\left(1+a_{1} t+\cdots\right) u_{0}+v_{1} t+\cdots \\
\left\|A_{i \eta \eta}\right\|^{2} & =\left\|A_{0}\right\|^{2}+\lambda_{1} t+\cdots
\end{aligned}
$$

as we had before, with $v_{1} \perp u_{0}$, etc.

We equate the coefficients of $t$ in the equation

$$
A_{-i \eta} A_{i \eta} u_{i \eta}=\left\|A_{i \eta}\right\|^{2} u_{i \eta}
$$

to obtain

$$
a_{1} A_{0}^{2} u_{0}+A_{0}^{2} v_{1}+A_{0} A_{1} u_{0}-A_{1} A_{0} u_{0}=a_{1}\left\|A_{0}\right\|^{2} u_{0}+\left\|A_{0}\right\|^{2} v_{1}+\lambda_{1} u_{0}
$$


which is the same as

$$
A_{0}^{2} v_{1}+A_{0} A_{1} u_{0}-\left\|A_{0}\right\| A_{1} u_{0}=\left\|A_{0}\right\|^{2} v_{1}+\lambda_{1} u_{0}
$$

If we take the scalar product of this last equation with $u_{0}$ we obtain $\lambda_{1}=0$. Hence the linear term in $\eta$ is zero in the expansion of $\left\|A_{\xi+i \eta}\right\|^{2}$. Consequently we have

$$
\left\|A_{\xi+i \eta}\right\|^{2}=\left\|A_{0}\right\|^{2}+2\left\|A_{0}\right\| Q(\xi, \xi)+Q_{1}(\xi, \eta)+Q_{2}(\eta, \eta)+O\left([|\xi|+|\eta|]^{3}\right)
$$

where $Q$ is the quadratic form of (2.13) and $Q_{1}, Q_{2}$ are quadratic forms. The inequality (2.14) follows easily from this last expression and the negative definiteness of $Q$, on using the Schwarz inequality.

We conclude the proof by showing how to obtain (2.9) for $t$ not an integer. We have

$$
\rho_{r}(y)=\frac{E_{x}\left[E_{V}\left\{\exp \left[\int_{0}^{t} \varepsilon V(s, X(s)) d s\right]\right\} \delta(X(t)-X(0)-y)\right]}{E_{x} E_{V}\left\{\exp \left[\int_{0}^{\prime} \varepsilon V(s, X(s)) d s\right]\right\}}
$$

Putting $N=[t]$, the integer part of $t$, we have then

$$
\rho_{t}(y) \leqslant C \frac{E_{x}\left[E_{V}\left\{\exp \left[\int_{0}^{N} \varepsilon V(s, X(s)) d s\right]\right\} \delta(X(t)-X(0)-y)\right]}{E_{x} E_{V}\left\{\exp \left[\int_{0}^{N} \varepsilon V(s, X(s)) d s\right]\right\}}
$$

for some constant $C$ independent of $x, y, t$. Next we write

$$
X(t)-X(0)-y=X(N)-X(0)-[y+Y]
$$

where $Y=X(N)-X(t)$ is independent now of $X(s), 0 \leqslant s \leqslant N$. Thus

$$
\rho_{r}(y) \leqslant C E\left[\rho_{N}(y+Y)\right]
$$

The inequality (2.9) follows now from the previous inequality, since $Y$ is Gaussian with mean zero and variance less than or equal to 1 . QED

Proposition 2.3. There exist $\eta>0$ and a Gaussian random variable $Y$ such that for any measurable function $f: \mathbb{R}^{d} \rightarrow \mathbb{R}$ with

$$
\int_{\mathbb{R}^{\prime \prime}} e^{-n|\cdot| \cdot \mid}|f(y)| d y<\infty
$$

then

$$
\lim _{t \rightarrow \infty} E_{x}^{A}\left[f\left(\frac{X(t)-X(0)}{\sqrt{t}}\right)\right]=E[f(Y)]
$$


Proof. Let us first assume that $f$ is a Schwartz function and that $t=N$ is an integer. Then we have

$$
E_{x}^{A}\left[f\left(\frac{X(t)-X(0)}{\sqrt{t}}\right)\right]=\int_{\mathbb{R}^{\prime \prime}} \phi_{N}\left(\frac{\xi}{\sqrt{N}}\right) \hat{f}(\xi) d \xi
$$

where $\phi_{N}$ is given by $(2.5)$ and $\hat{f}$ is the Fourier transform of $f$. We estimate the last integral by using the Poisson summation formula as in Proposition 2.2. Thus we have

$$
\begin{aligned}
\sum_{n \in \mathbb{Z}^{d}}\left\langle\chi_{\bar{\xi}+2 \pi n}(\bar{x}) \delta_{\bar{x}}, A_{\xi+2 \pi n}^{N} \chi_{\xi+2 \pi n}\right\rangle \hat{f}([\xi+2 \pi n] \sqrt{N}) \\
\quad=\left\langle\chi_{\bar{\xi}}(\bar{x}) \delta_{. \bar{x}}, A_{\xi}^{N}\left[\chi_{\xi} \sum_{n \in \mathbb{Z}^{\prime \prime}} \chi_{2 \pi n} \hat{f}([\xi+2 \pi n] \sqrt{N}) \exp [-2 \pi n i \cdot \bar{x}]\right]\right\rangle \\
=\left\langle\chi_{\bar{\xi}}(\bar{x}) \delta_{\bar{x}}, A_{\xi}^{N} \chi_{\xi}\right\rangle \hat{f}(\xi \sqrt{N})+E_{N}(\xi)
\end{aligned}
$$

when $E_{N}(\xi)$ is simply the remainder term. Hence

$$
\begin{aligned}
\int_{\mathbb{R}_{d}} \phi_{N}(\xi / \sqrt{N}) \hat{f}(\xi) d \xi \\
\quad=N^{d / 2} \int_{\mathbb{R}^{d}} \phi_{N}(\xi) \hat{f}(\xi \sqrt{N}) d \xi \\
\quad=\left[N^{d / 2} \int_{J} d \xi\left\{\left\langle\chi_{\xi}(\bar{x}) \delta_{\bar{x}}, A_{\xi}^{N}\right\rangle \hat{f}(\xi \sqrt{N})+E_{N}(\xi)\right\}\right]\left\langle\delta_{\bar{x}}, A_{0}^{N} \chi_{0}\right\rangle
\end{aligned}
$$

where $J=[-\pi, \pi]^{d}$. Since $\hat{f}$ is a Schwartz function, we have

$$
\begin{aligned}
\lim _{N \rightarrow \infty} & {\left[N^{d / 2} \int_{J} d \xi\left\langle\chi_{\bar{\xi}}(\bar{x}) \delta_{\bar{x}}, A_{\xi}^{N} \chi_{\bar{\xi}}\right\rangle \hat{f}(\xi \sqrt{N})\right]\left\langle\left\langle\delta_{\bar{x}}, A_{0}^{N} \chi_{0}\right\rangle\right.} \\
& \lim _{N \rightarrow \infty}\left[N^{d / 2} \int_{|\xi|<1 / N^{1 / 3}} d \xi\{\cdot\}\right]\left\langle\delta_{\bar{x}}, A_{0}^{N} \chi_{0}\right\rangle \\
\quad= & \lim _{N \rightarrow \infty}\left[N^{d / 2} \int_{|\xi|<1 / N^{1 / 3}} d \xi\left\langle\delta_{\bar{x}}, A_{\bar{\xi}}^{N} \chi_{0}\right\rangle \hat{f}(\xi \sqrt{N})\right] /\left\langle\delta_{\bar{x}}, A_{0}^{N} \chi_{0}\right\rangle
\end{aligned}
$$

We have now that for $|\xi|<1 / N^{1 / 3}$,

$$
\begin{aligned}
\left\langle\delta_{\tilde{x}}, A_{\xi}^{N} \chi_{0}\right\rangle & =\left\langle A_{\xi} \delta_{\tilde{x}}, A_{\xi}^{N-1} \chi_{0}\right\rangle \\
& =\left\langle A_{\xi} \delta_{\bar{x}}, u_{\xi}\right\rangle\left\langle u_{\xi}, \chi_{0}\right\rangle\left\|A_{\xi}\right\|^{N-1}\left[1+O\left(e^{-\delta N}\right)\right]
\end{aligned}
$$


for some $\delta>0$. Here $u_{\xi}$ is the principal eigenvector of $A_{\xi}$ and $\delta$ is related to the spectral gap of $A_{\bar{\zeta}}$ as given by Proposition 2.1(d). We therefore have

$$
\begin{aligned}
\left\langle\delta_{\bar{x}},\right. & \left.A_{\xi}^{N} \chi_{0}\right\rangle /\left\langle\delta_{\bar{x}}, A_{0}^{N} \chi_{0}\right\rangle \\
& =\left[1+O\left(1 / N^{1 / 3}\right)\right]\left\|A_{\tilde{\xi}}\right\|^{N-1} /\left\|A_{0}\right\|^{N-1}, \quad|\xi|<1 / N^{1 / 3}
\end{aligned}
$$

Using (2.13) and the previous identity, we conclude that

$$
\begin{aligned}
\lim _{N \rightarrow \infty} & {\left[N^{d / 2} \int_{|\xi|<1 / N^{1 / 3}} d \xi\left\langle\delta_{\bar{x}}, A_{\tilde{\xi}}^{N} \chi_{0}\right\rangle \hat{f}(\xi \sqrt{N})\right]\left\langle\left\langle\delta_{\bar{x}}, A_{0}^{N} \chi_{0}\right\rangle\right.} \\
& \left.=\lim _{N \rightarrow \infty} N^{d / 2} \int_{|\xi|<1 / N^{1 / 3}} d \xi \exp \left[N Q(\xi, \xi) /\left\|A_{0}\right\|\right] \hat{f}(\xi \sqrt{N})\right)
\end{aligned}
$$

where $Q$ is the quadratic form in (2.13). Since $Q$ is negative definite, this last limit is the same as

$$
\int_{\mathbb{R}^{d}} d \xi \exp \left[Q(\zeta, \xi) /\left\|A_{0}\right\|\right] \hat{f}(\xi)=E[f(Y)]
$$

where $Y$ is a Gaussian random variable.

Observe next that the remainder term $E_{N}(\xi)$ satisfies the inequality

$$
\sup _{\xi \in J}\left|E_{N}(\xi)\right| /\left\langle\delta_{\bar{x}}, A_{0}^{N} \chi_{0}\right\rangle \leqslant C_{k} / N^{k}
$$

where $k$ is an arbitrary positive integer and $C_{k}$ is independent of $N$. This follows from the fact that $\hat{f}$ is a Schwartz function. Proposition 2.3 for integer $t$ and Schwartz functions $f$ immediately follows.

We extend the result to noninteger $t$ and Schwartz functions $f$. To do this put $N=[t]$ and

$$
Y=\frac{X(t)-X(0)}{\sqrt{t}}-\frac{X(N)-X(0)}{\sqrt{N}}
$$

Then we have

$$
f\left(\frac{X(t)-X(0)}{\sqrt{t}}\right)=f\left(\frac{X(N)-X(0)}{\sqrt{N}}\right)+Y \cdot \int_{0}^{1} d s \nabla f\left(\frac{X(N)-X(0)}{\sqrt{N}}+s Y\right)
$$


Just as in Proposition 2.2, we have

$$
\begin{aligned}
& E_{x}^{A}\left[|Y| \int_{0}^{1} d s|\nabla f|\left(\frac{X(N)-X(0)}{\sqrt{N}}+s Y\right)\right] \\
& \quad \leqslant C E\left[|Y| \int_{0}^{1} d s \int_{\mathbb{R}^{\prime}}|\nabla f|\left(\frac{y}{\sqrt{N}}+s Y\right) \rho_{N}(y) d y\right]
\end{aligned}
$$

where $Y$ is the Gaussian random variable defined by (2.15) under the assumption that $X$ is Brownian motion. It is evident that the mean of $Y$ is bounded by $C_{1} / N^{3 / 2}$ and the variance by $C_{2} / N$ for constants $C_{1}, C_{2}$. If we use Proposition 2.2 to estimate $\rho_{N}(y)$, then we have

$$
\begin{aligned}
& E\left[|Y| \int_{0}^{1} d s \int_{\mathbb{R}^{d}}|\nabla f|\left(\frac{y}{\sqrt{N}}+s Y\right) \rho_{N}(y) d y\right] \\
& \leqslant C E\left[|Y| \int_{0}^{1} d s \int_{\mathbb{R}^{d}} \nabla f(z+s Y) \exp (-c|z|) d z\right]
\end{aligned}
$$

for positive constants $C, c>0$. Since the mean and variance of $Y$ are $O(1 / N)$, this last expression is $O\left(1 / N^{1 / 2}\right)$. Hence the result for noninteger $t$ follows from the integer- $t$ result.

Finally we wish to extend the class of functions $f$ from Schwartz space to the class given in the statement of the proposition. This follows immediately from Proposition 2.2. In fact for any $\delta>0$ there is a Schwartz space function $g$ such that

$$
\int_{\mathbb{R}^{d}} e^{-\eta|\cdot|}|f(y)-g(y)| d y<\delta
$$

It follows from (2.16) and Proposition 2.2 that if $\eta>0$ is sufficiently small, then

$$
\sup _{t \geqslant 1} E_{x}^{A}\left[\left|f\left(\frac{X(t)-X(0)}{\sqrt{t}}\right)-g\left(\frac{X(t)-X(0)}{\sqrt{t}}\right)\right|\right]<C \delta
$$

from some constant $C>0$. The result now follows from this last inequality by standard argument. QED

We have shown in Proposition 2.3 that the process $X(t)$ with measure (1.7) is in some sense a renormalized Brownian motion. Here we wish to 
show that it has similar recurrence properties to Brownian motion. Let us consider two independent copies $X(t), Y(t)$ of the process (1.7). Thus the pair $(X(t), Y(t))$ forms a process in $\mathbb{R}^{2 d}$. We consider a subset $\mathscr{F}$ of $\mathbb{R}^{2 d}$ defined by

$$
\mathscr{S}=\bigcup\left\{Q_{n} \times Q_{n}: n \in \mathbb{Z}^{d}\right\}
$$

where as before $Q_{n}$ is the unit cube centered at $n \in \mathbb{Z}^{d}$. Now the dimension of the set $\mathscr{S}$ is $d$. Hence by the recurrence properties of Brownian motion we should expect that for $d \geqslant 3$ the process $(X(t), Y(t))$ spends a finite amount of time in $\mathscr{S}$. We shall prove a version of the statement as follows:

Theorem 2.1. Let $\chi_{\mathscr{Y}}$ be the characteristic function of the set $\mathscr{S}$. Then there exist $\delta_{0}, \alpha>0$, and a constant $C$ sufficiently large such that

$$
\begin{aligned}
& \frac{1}{|\Omega|} \int_{\Omega} E_{x, y}^{A}\left[\exp \left[\delta \int_{0}^{t} \chi_{S}(X(s), Y(s)) d s\right]\right] d x d y \\
& \quad \leqslant 1+\frac{C \delta}{(1+|\Omega|)^{\alpha}}, \quad t \geqslant 0
\end{aligned}
$$

for any $\delta \leqslant \delta_{0}$ and ball $\Omega \subset \mathbb{R}^{2 d}, d \geqslant 3$.

Proof. We write the expression on the left in (2.7) as a sum

$$
\sum_{n=0}^{\infty} \delta^{n} a_{n}
$$

where $a_{0}=1$ and $a_{n}$ for $n \geqslant 1$ is given by the formula

$$
a_{n}=\frac{1}{|\Omega|} \int_{\Omega} E_{x, y}^{A}\left[\int_{0<s_{1}<s_{2}<\cdots<s_{n}<1} d s_{1} \cdots d s_{n} \prod_{i=1}^{n} \chi_{\mathscr{S}}\left(X\left(s_{i}\right), Y\left(s_{i}\right)\right)\right] d x d y
$$

We can rewrite $a_{n}$ in terms of the joint probability density

$$
(X(0), Y(0)),\left(X\left(s_{1}\right), Y\left(s_{1}\right)\right), \ldots,\left(X\left(s_{n}\right), Y\left(s_{n}\right)\right)
$$

which we denote by

$$
\rho_{l}\left(x, y, 0 ; x_{1}, y_{1}, s_{1} ; x_{2}, y_{2}, s_{2} ; \ldots x_{n}, y_{n}, s_{n}\right)
$$


as

$$
\begin{aligned}
a_{n}= & \frac{1}{|\Omega|} \int_{\Omega} d x d y \int_{\mathscr{S}^{\prime}} d x_{1} d y_{1} \int_{\mathscr{Y}^{\prime}} d x_{2} d y_{2} \ldots \int_{\mathscr{S}^{\prime}} d x_{n} d y_{n} \\
& \times \int_{0<s_{1}<s_{2}<\cdots<s_{n}<t} d s_{1} \ldots d s_{n} \\
& \times \rho_{r}\left(x, y, 0 ; x_{1}, y_{1}, s_{1} ; x_{2}, y_{2}, s_{2} ; \ldots x_{n}, y_{n}, s_{n}\right)
\end{aligned}
$$

Next we use the following proposition, which proves

$$
\begin{aligned}
& p_{t}\left(x, y, 0 ; x_{1}, y_{1}, s_{1} ; x_{2}, y_{2}, s_{2} ; \ldots x_{n}, y_{n}, s_{n}\right) \\
& \leqslant \frac{C_{1}^{n}}{s_{1}^{d}} \exp \left(\frac{-c_{2}\left(\left|x_{1}-x\right|+\left|y_{1}-y\right|\right)}{s_{1}^{1 / 2}}\right) \\
& \times \prod_{i=1}^{n-1} \frac{1}{\left(s_{i+1}-s_{i}\right)^{d}} \exp \left(\frac{-c_{2}\left(\left|x_{i+1}-x_{i}\right|+\left|y_{i+1}-y_{i}\right|\right)}{\left(s_{i+1}-s_{i}\right)^{1 / 2}}\right)
\end{aligned}
$$

for some constants $C_{1}, c_{2}>0$ independent of $x, y, t$ and the $x_{i}, y_{i}, s_{i}$, $1 \leqslant i \leqslant n$. Using the fact that

$$
\int_{0}^{\infty} d s \frac{1}{s^{d}} \exp \left(\frac{-c_{2}(|x|+|y|)}{s^{1 / 2}}\right) \leqslant \frac{C_{3}}{(|x|+|y|)^{2 d-2}}
$$

for some constant $C_{3}$ depending only on $c_{2}$ and the fact that

$$
\int_{y^{\prime}} d x_{i+1} d y_{i+1} /\left(\left|x_{i}-x_{i+1}\right|+\left|y_{i}-y_{i+1}\right|\right)^{2 d-2} \leqslant C_{4}<\infty
$$

where the constant $C_{4}$ is independent of $\left(x_{i}, y_{i}\right)$, we conclude

$$
a_{n} \leqslant\left(C_{5}^{n} /|\Omega|\right) \int_{\Omega} d x d y \int_{\mathscr{G}} d x_{1} d y_{1} /\left(\left|x-x_{1}\right|+\left|y-y_{1}\right|\right)^{2 d-2}
$$

for some fixed constant $C_{5}>0$. Observe that the RHS of the last inequality is bounded by a constant times $C_{5}^{n}$. To get the decay in $|\Omega|$ as $|\Omega| \rightarrow \infty$, let $\Omega_{j}$ be the ball concentric with $\Omega$ which has radius equal to $2^{j}$ times the radius of $\Omega, j=0,1,2, \ldots$. 
Then we can write

$$
\begin{aligned}
& (1 /|\Omega|) \int_{\Omega} d x d y \int_{\mathscr{S}} d x_{1} d y_{1} /\left(\left|x-x_{1}\right|+\left|y-y_{1}\right|\right)^{2 d-2} \\
& =(1 /|\Omega|) \int_{\Omega} d x d y \int_{\mathscr{S} \cap \Omega} d x_{1} d y_{1} /\left(\left|x-x_{1}\right|+\left|y-y_{1}\right|\right)^{2 d-2} \\
& \quad+\sum_{j=0}^{\infty} \frac{1}{|\Omega|} \int_{\Omega} d x d y \int_{\mathscr{S} \cap\left(\Omega_{j+1} \backslash \Omega_{j}\right)} d x_{1} d y_{1} /\left(\left|x-x_{1}\right|+\left|y-y_{1}\right|\right)^{2 d-2}
\end{aligned}
$$

Now if we take a unit cube $Q \subset \mathbb{R}^{d}$ with $Q \times Q \subset \mathscr{S} \cap \Omega$, then it is clear that

$$
(1 /|\Omega|) \int_{\Omega} d x d y \int_{Q \times Q} d x_{1} d y_{1} /\left(\left|x-x_{1}\right|+\left|y-y_{1}\right|\right)^{2 d-2} \leqslant C /|\Omega|^{1-1 / d}
$$

for some constant $C$. Since $\mathscr{S} \cap \Omega$ is the union of order- $|\Omega|^{1 / 2}$ unit cubes, we conclude that

$$
(1 /|\Omega|) \int_{\Omega} d x d y \int_{\mathscr{S} \cap \Omega} d x_{1} d y_{1} /\left(\left|x-x_{1}\right|+\left|y-y_{1}\right|\right)^{2 d-2} \leqslant C /|\Omega|^{1 / 2-1 / d}
$$

Hence we have bounded the first term on the RHS of (2.19) appropriately. Clearly we have a similar bound for the $j=0$ term also. The $j$ th term in the sum for $j \geqslant 1$ is bounded by $C / 2^{j(d-2)}|\Omega|^{1 / 2-1 / d}$. Hence, on summing the series in $(2.19)$, we conclude that

$$
a_{n} \leqslant C_{5}^{n} C_{6} /(1+|\Omega|)^{\alpha}
$$

where $C_{6}$ is a constant and $\alpha=1 / 2-1 / d$. Now if we choose $\delta_{0}$ such that $C_{5} \delta_{0}<1$, the inequality (2.17) follows. QED

We are left to prove (2.18). This is evidently a generalization of Proposition 2.2. First observe that since the processes $X, Y$ are independent, it will be sufficient to consider $X$ alone. The inequality (2.18) follows then from the next result.

Proposition 2.4. Let $0=s_{0}<s_{1}<s_{2}<\cdots<s_{n}<t$ and $x_{i} \in \mathbb{R}^{d}$, $0 \leqslant i \leqslant n$. Suppose $\rho_{1}\left(x_{0}, s_{0}, x_{1}, s_{1}, \ldots, x_{n}, s_{n}\right)$ is the joint density of the variables $X\left(s_{1}\right), X\left(s_{2}\right), \ldots, X\left(s_{n}\right)$ for the averaged process (1.7) started at $x_{0}$ at time 0 . Then there is a constant $C>0$ such that

$$
\rho_{l}\left(x_{0}, s_{0}, x_{1}, s_{1}, \ldots, x_{n}, s_{n}\right) \leqslant C^{\prime \prime} \prod_{i=1}^{n} \rho_{s_{i}-s_{i-1}}\left(x_{i-1}, s_{i-1}, x_{i}, s_{i}\right)
$$


Proof. The inequality (2.20) with $C=1$ would follow from (1.7) if the normalization constant in (1.7) were unity. However, the normalization constant has the form $\gamma\left\|A_{0}\right\|^{\prime}$, where $\gamma$ depends on $t$ and also on the starting point and time. However, there are constants $c_{1}, C_{1}>0$ such that $c_{1} \leqslant \gamma \leqslant C_{1}$ for all starting points and times $t$. The constant $C$ in $(2.20)$ is then bounded by $C \leqslant C_{1} / c_{1}$.

\section{THE PARTITION FUNCTION}

Here we establish some properties of the partition function defined by (1.5). Now $Z_{x, v}(t)$ is a random variable in $V$ and it is clear that it has expectation value equal to 1 . Thus by the Chebyshev inequality one has for any given $t$ and $\alpha>0$ the inequality

$$
P\left(Z_{x, v}(t)>\alpha\right) \leqslant 1 / \alpha
$$

We shall prove the following:

Proposition 3.1. There is a constant $C>0$ such that for any $\alpha>0$ there is the inequality

$$
P\left(\sup _{t>0} Z_{x, V}(t)>\alpha\right) \leqslant C / \alpha
$$

Proof. Let $\tau$ be the smallest integer such that

$$
\sup _{0<i<\tau} Z_{x, V}(t)>\alpha
$$

In particular, we must have $Z_{x, v}(t) \geqslant \alpha$ for some $t$ with $\tau-1<t \leqslant \tau$. Now let $N$ be a large integer. Then we have

$$
1=E\left[Z_{x, V}(N)\right]=E\left\{E\left[Z_{x, V}(N) \mid V(s, x), s \leqslant \tau \wedge N, x \in \mathbb{R}^{d}\right]\right\}
$$

It is clear now from the previous section that there is a constant $c_{1}>0$ such that

$$
E\left[Z_{x, v}(N) \mid V(s, x), s \leqslant \tau \wedge N, x \in \mathbb{R}^{d}\right] \geqslant c_{1} Z_{x, v}(\tau \wedge N)
$$

Hence we conclude that

$$
E\left[Z_{x, \nu}(\tau \wedge N)\right] \leqslant 1 / c_{1}
$$

If $\tau<N$, then there is a constant $c_{2}$ such that $Z_{x, v}(\tau \wedge N) \geqslant c_{2} \alpha$, and so we have

$$
c_{2} \alpha P(\tau<N) \leqslant 1 / c_{1}
$$

which yields the result. QED 
Observe that Proposition 3.1 holds for all $\varepsilon>0$ and all dimensions $d$. In contrast, the behavior of $\inf _{r>0} Z_{x, v}(t)$ appears to depend critically on both $\varepsilon$ and $d$.

Proposition 3.2. Let $\delta$ satisfy $0<\delta<1 / 2$. Then there exists $\varepsilon_{0}>0$ such that if $\varepsilon \leqslant \varepsilon_{0}$ and $d \geqslant 3$, there are constants $C, \alpha>0$ depending only on $d$ such that

$$
P\left(\inf _{1>0} Z_{x, V}(t)<\delta\right) \leqslant C /|\log \delta|^{\alpha}
$$

Proof. Let $\tau$ be the smallest integer such that

$$
\inf _{0<i<\tau} Z_{x, v}(t)<\delta
$$

As in Proposition 3.1, we must have $Z_{x, v}(t)<\delta$ for some $t$ with $\tau-1<$ $t \leqslant \tau$. Let $N$ be a larger integer. Then there is a constant $C_{1}>0$ such that

$$
E\left[Z_{x, v}(N) \mid V(s, x), s \leqslant \tau \wedge N, x \in \mathbb{R}^{d}\right] \leqslant C_{1} Z_{x, V}(\tau \wedge N)
$$

Hence we have the inequality

$$
1 \leqslant C_{2} \delta P(\tau<N)+E\left[Z_{x, V}(N) ; \tau \geqslant N\right]
$$

where $C_{2}$ is a constant.

We have now by the Schwarz inequality

$$
E\left[Z_{x, V}(N) ; \tau \geqslant N\right] \leqslant P(\tau \geqslant N)^{1 / 2} E\left[Z_{x, V}(N)^{2}\right]^{1 / 2}
$$

Hence if we put

$$
M=\sup _{N \geqslant 1} E\left[Z_{x, v}(N)^{2}\right]
$$

and assume $M$ is finite, then we have on letting $N \rightarrow \infty$ in (3.2) the inequality

$$
1 \leqslant C_{2} \delta P(\tau<\infty)+M^{1 / 2}[1-P(\tau<\infty)]^{1 / 2}
$$

If $C_{2} \delta<1$, it follows from this last inequality that

$$
P(\tau<\infty) \leqslant 1-\left(1-C_{2} \delta\right)^{2} / M
$$

Hence if $M<\infty$, we see that the LHS of (3.1) is strictly less than 1 for small $\delta$. However, the inequality (3.3) does not give a dependence on $\delta$ as $\delta \rightarrow 0$. 
We shall show now that $M<\infty$. From (1.15) we have

$$
\begin{aligned}
E\left[Z_{x, V}(N)^{2}\right]= & E_{x, x}^{A}\left[E_{V}\left\{\exp \left[\int_{0}^{N} \varepsilon V(s, X(s))+\varepsilon V(s, Y(s)) d s\right]\right\}\right. \\
& \times\left(E_{V}\left\{\exp \left[\int_{0}^{N} \varepsilon V(s, X(s)) d s\right]\right\}\right. \\
& \left.\left.\times E_{V}\left\{\exp \left[\int_{0}^{N} \varepsilon V(s, Y(s)) d s\right]\right\}\right)^{-1}\right]
\end{aligned}
$$

where $E_{x, x}^{A}$ denotes that the expectation is with respect to the averaged process (1.7), and $X, Y$ are two independent copies of the process. Now for $i=1, \ldots, n \in \mathbb{Z}^{d}$ let $\tau_{i, n}(X)$ be the amount of time the path $X(s)$ spends in the cube $Q_{n}$ during the time interval $i-1 \leqslant s \leqslant i$. Similarly we define $\tau_{i, n}(Y)$. Then it is clear that

$$
\exp \left[\int_{0}^{N} \varepsilon V(s, X(s)) d s\right]=\exp \left[\sum_{i=1}^{N} \sum_{n \in \mathbb{Z}^{d}} \varepsilon V_{i, n} \tau_{i, n}(X)\right]
$$

whence

$$
E_{V}\left[\exp \left[\int_{0}^{N} \varepsilon V(s, X(s)) d s\right]\right]=\prod_{i=1}^{N} \prod_{n \in \mathbb{Z}^{d}} \cosh \left(\varepsilon \tau_{i, n}(X)\right)
$$

Consequently, we have

$$
\begin{aligned}
& \frac{E_{V}\left\{\exp \left[\int_{0}^{N} \varepsilon V(s, X(s))+\varepsilon V(s, Y(s)) d s\right]\right\}}{E_{V}\left\{\exp \left[\int_{0}^{N} \varepsilon V(s, X(s)) d s\right]\right\} E_{V}\left\{\exp \left[\int_{0}^{N} \varepsilon V(s, Y(s)) d s\right]\right\}} \\
& =\prod_{i=1}^{N} \prod_{n \in \mathbb{Z}^{d}} \frac{\cosh \left[\varepsilon \tau_{i, n}(X)+\varepsilon \tau_{i, n}(Y)\right]}{\cosh \left[\varepsilon \tau_{i, n}(X)\right] \cosh \left[\varepsilon \tau_{i, n}(Y)\right]} \\
& \leqslant \prod_{i=1}^{N} \prod_{n \in \mathbb{Z}^{d}}\left[1+\varepsilon^{2} \tau_{i, n}(X) \tau_{i, n}(Y)\right] \\
& \leqslant \exp \left[\sum_{i=1}^{N} \sum_{n \in \mathbb{Z}^{d}} \varepsilon^{2} \tau_{i, n}(X) \tau_{i, n}(Y)\right] \\
& =\exp \left[\sum_{i=1}^{N} \sum_{n \in \mathbb{Z}^{d}} \varepsilon^{2} \int_{i-1}^{i} \chi_{Q_{n}}(X(s)) d s \int_{i-1}^{i} \chi_{Q_{n}}(Y(s)) d s\right]
\end{aligned}
$$




$$
\begin{aligned}
& \leqslant \exp \left[\sum_{n \in \mathbb{Z}^{d}} \int_{0}^{1} \varepsilon^{2} \int_{0}^{N} \chi_{Q_{n}}(X(s)) \chi_{Q_{n}}(Y(s+u))\right. \\
& \left.\quad+\chi_{Q_{n}}(X(s+u)) \chi_{Q_{n}}(Y(s)) d s d u\right] \\
& =\exp \left[\int_{0}^{1} \varepsilon^{2} \int_{0}^{N} \chi_{\mathscr{S P}}(X(s), Y(s+u))+\chi_{\mathscr{P}}(X(s+u), Y(s)) d s d u\right]
\end{aligned}
$$

where $\mathscr{S}=\bigcup\left\{Q_{n} \times Q_{n}: n \in \mathbb{Z}^{d}\right\}$ is the set defined prior to Theorem 2.1. Hence we have

$$
\begin{aligned}
& E\left[Z_{x, V}(N)^{2}\right] \\
& \leqslant E_{x, x}^{A}\left[\exp \left[\int_{0}^{1} \varepsilon^{2} \int_{0}^{N} \chi_{\mathscr{S}}(X(s), Y(s+u))+\chi_{\mathscr{S}}(X(s+u), Y(s)) d s d u\right]\right] \\
& \leqslant \int_{0}^{1} d u E_{x, x}^{A}\left[\exp \left[\varepsilon^{2} \int_{0}^{N} \chi_{S,}(X(s), Y(s+u))+\chi_{\mathscr{S}}(X(s+u), Y(s)) d s\right]\right] \\
& \leqslant \frac{1}{2} \int_{0}^{1} d u\left\{E_{x, x}^{A}\left[\exp \left[2 \varepsilon^{2} \int_{0}^{N} \chi_{\mathscr{S}}(X(s), Y(s+u)) d s\right]\right]\right. \\
& \left.\quad+E_{x, x}^{A}\left[\exp \left[2 \varepsilon^{2} \int_{0}^{N} \chi_{S_{S}}(X(s+u), Y(s)) d s\right]\right]\right\}
\end{aligned}
$$

by Jensen's inequality. Theorem 2.1 now tells us that the RHS of this last inequality is uniformly bounded as $N \rightarrow \infty$ provided $\varepsilon$ is sufficiently small. Hence $M$ is finite.

Next we wish to obtain the dependence in $\delta$ for the RHS of (3.1). To do this we define for $k=1,2, \ldots$ a partition function $Z_{x, V, k}(t)$ by

$$
Z_{x, V, k}(t)=\frac{E_{x}\left\{\exp \left[\int_{k}^{t} \varepsilon V(s, X(s)) d s\right]\right\}}{E_{V} E_{x}\left\{\exp \left[\int_{k}^{t} \varepsilon V(s, X(s)) d s\right]\right\}}, \quad t \geqslant k
$$

It is clear that there is a number $a>0$ such that

$$
Z_{x, V}(t) \geqslant a^{k} Z_{x, V, k}(t), \quad t \geqslant k
$$

Evidently we have

$$
E\left[Z_{x, V, k}(t)\right]=1, \quad k \leqslant t
$$


On the other hand,

$$
\begin{aligned}
E\left[Z_{x, V, k}(t)^{2}\right] \leqslant & \int_{\mathbb{R}^{\prime \prime}} \int_{\mathbb{P}^{d}} d z d w \rho(z, w) \\
& \times \frac{1}{2} \int_{0}^{1} d u\left\{E_{z, w}^{A}\left[\exp \left[2 \varepsilon^{2} \int_{0}^{t-k} \chi_{S P}(X(s), Y(s+u)) d s\right]\right]\right. \\
& \left.+E_{z, w}^{A}\left[\exp \left[2 \varepsilon^{2} \int_{0}^{t-k} \chi_{S P}(X(s+u), Y(s)) d s\right]\right]\right\}
\end{aligned}
$$

where the density $\rho(z, w)$ is given by

$$
\begin{aligned}
\rho(z, w)= & \rho_{0}(z, w) E_{V} E_{z}\left[\exp \left[\int_{0}^{t-k} \varepsilon V(s, X(s)) d s\right]\right] \\
& \times E_{V} E_{w}\left[\exp \left[\int_{0}^{t-k} \varepsilon V(s, Y(s)) d s\right]\right] / \text { normalization }
\end{aligned}
$$

where $\rho_{0}$ is the density for Brownian motion in $\mathbb{R}^{2 d}$ at time $k$ started at $(x, x)$ at time 0 . Clearly $\rho(z, w)$ is concentrated on a scale of radius $k^{1 / 2}$. Hence by Theorem 2.1 we have

$$
E\left[Z_{x, V . k}(t)^{2}\right] \leqslant 1+C \varepsilon^{2} / k^{d x}, \quad t \geqslant k
$$

provided $\varepsilon$ is sufficiently small and again the bound is uniform in $t$ as $t \rightarrow \infty$.

Now for $k=1,2, \ldots$ let

$$
M_{k}=\sup _{N \geqslant k} E\left[Z_{x, V, k}(N)^{2}\right]
$$

and $\tau_{k}$ be the smallest integer $\geqslant k$ such that

$$
\inf _{k \leqslant i<\tau_{k}} Z_{x, V, k}(t)<\delta
$$

As before, we have

$$
\begin{gathered}
E\left[Z_{x, V, k}(N) \mid V(s, x), s \leqslant \tau_{k} \wedge N, x \in \mathbb{R}^{d}\right] \\
\leqslant C_{1} Z_{x, V, k}\left(\tau_{k} \wedge N\right), \quad N>k
\end{gathered}
$$

Hence we have an inequality analogous to (3.3), namely

$$
P\left(\tau_{k}<\infty\right) \leqslant 1-\left(1-C_{2} \delta\right)^{2} / M_{k}
$$


Using the inequality (3.7), we have that

$$
\begin{aligned}
P\left(\tau_{k}<\infty\right) & \leqslant 1-1 / M_{k}+2 C_{2} \delta \\
& \leqslant C \varepsilon^{2} / k^{d \alpha}+2 C_{2} \delta
\end{aligned}
$$

If we take $\delta=\varepsilon^{2} / k^{d \alpha}$, we conclude that

$$
P\left(\inf _{t \geqslant k} Z_{x, V, k}(t)<\varepsilon^{2} / k^{d \alpha}\right) \leqslant C \varepsilon^{2} / k^{d \alpha}
$$

Hence from (3.6) it follows that

$$
P\left(\inf _{i>0} Z_{x, V}(t)<\varepsilon^{2} a^{k} / k^{d \alpha}\right) \leqslant C \varepsilon^{2} / k^{d \alpha}
$$

The inequality (3.1) follows immdiately from this last inequality by choosing $k$ appropriately, depending on $\delta$. QED

Next we wish to address the problem of showing that $Z_{x, V}(t)$ converges to a limit as $t \rightarrow \infty$. We shall show that, provided we take an exponentially growing sequence of $t$ values, the limit exists if $\varepsilon$ is small and $d \geqslant 3$. We are unable to make any statement about the convergence properties of $Z_{x, V}(t)$ if either $\varepsilon$ is large or $d \leqslant 2$. However, there is a quantity closely related to $Z_{x, V}(t)$ which converges for all $\varepsilon$ and dimension $d$. Let $u_{0}(x), x \in Q_{0}$, be the principal eigenvector of the operator $A_{0}$ of Section 2 with eigenvalue $\left\|A_{0}\right\|$. The function $u_{0}$ can be extended to all of $\mathbb{R}^{d}$ by translation. Thus for $x \in \mathbb{R}^{d}, u_{0}(x)=u_{0}(\bar{x})$, where $\bar{x} \in Q_{0}$ and $\bar{x}-x \in \mathbb{Z}^{d}$. For $N=1,2, \ldots$ we define $\bar{Z}_{x, v}(N)$ by

$$
\bar{Z}_{x, V}(N)=\frac{E_{x}\left[\exp \left[\int_{0}^{N} \varepsilon V(s, X(s)) d s\right] u_{0}(X(N))\right]}{E_{V} E_{x}\left[\exp \left[\int_{0}^{N} \varepsilon V(s, X(s)) d s\right] u_{0}(X(N))\right]}
$$

It follows from (2.4) that the denominator of the last expression is simply $\left\|A_{0}\right\|^{N} u_{0}(x)$. It is easy to verify that the sequence of random variables $\bar{Z}_{x, v}(N), N=1,2, \ldots$, is a martingale and

$$
E\left[\bar{Z}_{x, V}(N)\right]=1, \quad N=1,2, \ldots
$$

Hence by the martingale convergence theorem ${ }^{(2)}$ the $\operatorname{limit}_{N \rightarrow \infty} \bar{Z}_{x, V}(N)$ exists with probability 1 . This is true for all $\varepsilon>0$ and dimension $d$. Our convergence theorem for $Z_{x, v}(t)$ is based on the following: 
Proposition 3.3. There exists $\varepsilon_{0}>0$ such that if $\varepsilon \leqslant \varepsilon_{0}$ and $d \geqslant 3$, then

$$
E\left[\left\{Z_{x, v}(M)-Z_{x, \nu}(N)\right\}^{2}\right] \leqslant C / N^{\alpha}
$$

for any integers $N, M, 1 \leqslant N \leqslant M$. The constant $C$ and index $\alpha>0$ depend only on $\varepsilon_{0}$ and $d$.

Proof. Let $X(s), Y(s), s \geqslant 0$, be independent Brownian paths. For $i=1,2, \ldots$, we define $g_{i}(X, Y)$ by

$$
\begin{aligned}
g_{i}(X, Y)= & E_{V}\left[\exp \left[\varepsilon \int_{i-1}^{i} V(s, X(s))+V(s, Y(s)) d s\right]\right] \\
& \times\left\{E_{V}\left[\exp \left[\varepsilon \int_{i-1}^{i} V(s, X(s)) d s\right]\right]\right. \\
& \times E_{V}\left[\exp \left[\varepsilon \int_{i-1}^{i} V(s, Y(s)) d s\right]\right\}^{-1}
\end{aligned}
$$

Then according to Eq. (3.4) one has

$$
E\left[Z_{x, \nu}(N)^{2}\right]=E_{x, N}^{A, N, N}\left[\prod_{i=1}^{N} g_{i}(X, Y)\right]
$$

The right-hand side in the last equation is an expectation with respect to the averaged process (1.7). We have introduced the parameters $N, N$ to indicate the dependence of the averaged process on the time $t$. Similarly we have the expressions

$$
\begin{array}{r}
E\left[Z_{x, V}(M)^{2}\right]=E_{x, .,}^{A . M, M}\left[\prod_{i=1}^{M} g_{i}(X, Y)\right] \\
E\left[Z_{x, V}(M) Z_{x, V}(N)\right]=E_{x, .,}^{A, M, N}\left[\prod_{i=1}^{N} g_{i}(X, Y)\right]
\end{array}
$$

Letting $[\cdot]$ denote integer part, we have that

$$
\begin{aligned}
& \left|E_{x, N}^{A, N, N}\left[\prod_{i=1}^{N} g_{i}(X, Y)\right]-E_{x, X}^{A, N, N}\left[\prod_{i=1}^{[N / 2]} g_{i}(X, Y)\right]\right| \\
& \quad \leqslant E_{x, x}^{A, N, N}\left[\left[\prod_{i=1}^{[N / 2]} g_{i}(X, Y)\left|\prod_{i=[N / 2]+1}^{N} g_{i}(X, Y)-1\right|\right]\right. \\
& \quad \leqslant E_{x, x}^{A, N, N}\left[\left(\prod_{i=1}^{[N / 2]} g_{i}(X, Y)\right)^{2}\right]^{1 / 2} E_{x, x}^{A, N, N}\left[\left|\prod_{i=[N / 2]+1}^{N} g_{i}(X, Y)-1\right|^{2}\right]^{1 / 2}
\end{aligned}
$$


It follows now from (3.5) and Theorem 2.1 that

$$
\left|E_{x, N}^{A, N, N}\left[\prod_{i=1}^{N} g_{i}(X, Y)\right]-E_{X, N}^{A, N, N}\left[\prod_{i=1}^{[N / 2]} g_{i}(X, Y)\right]\right| \leqslant C / N^{\alpha}
$$

for suitable constants $C, \alpha>0$. Using the formulas (3.8) and arguing as above, we conclude there are positive constants $C, \alpha$ such that

$$
\begin{aligned}
& E\left[\left\{Z_{x, V}(M)-Z_{x, V}(N)\right\}^{2}\right] \\
& \leqslant E_{x, x}^{A, N, N}\left[\prod_{i=1}^{[N / 2]} g_{i}(X, Y)\right] \\
& \quad+E_{x, X}^{A, M, M}\left[\prod_{i=1}^{[N / 2]} g_{i}(X, Y)\right]-2 E_{x, x}^{A, M, N}\left[\prod_{i=1}^{[N / 2]} g_{i}(X, Y)\right]+C / N^{\alpha}
\end{aligned}
$$

Next let us define a function $w(z)$ for $z \in Q_{0}$ by

$$
w(z)=A_{0}^{N-[N / 2]} \chi_{0}(z) A_{0}^{[N / 2]} \chi_{0}(x) / A_{0}^{N} \chi_{0}(x)
$$

The function $w$ is extended to all of $\mathbb{R}^{d}$ by periodicity, $w(z+n)=w(z)$, $n \in \mathbb{Z}^{d}$. Then we have

$$
\begin{aligned}
E_{x, X}^{A, N, N} & {\left[\prod_{i=1}^{[N / 2]} g_{i}(X, Y)\right] } \\
= & E_{x, X}^{A,[N / 2],[N / 2]}\left[\prod_{i=1}^{[N / 2]} g_{i}(X, Y) w(X([N / 2])) w(Y([N / 2]))\right]
\end{aligned}
$$

It is clear from Proposition 2.1 that

$$
w(z)=\left\langle\chi_{0}, u_{0}\right\rangle u_{0}(z)+O\left(e^{-c N}\right)
$$

for some positive constant $c$. Hence

$$
\begin{aligned}
E_{x, X}^{A, N . N} & {\left[\prod_{i=1}^{[N / 2]} g_{i}(X, Y)\right] } \\
= & E_{x, X}^{A,[N / 2],[N / 2]}\left[\prod_{i=1}^{[N / 2]} g_{i}(X, Y)\right. \\
& \left.\quad \times\left\langle\chi_{0}, u_{0}\right\rangle^{2} u_{0}(X([N / 2])) u_{0}(Y([N / 2]))\right]+O\left(e^{-c N}\right)
\end{aligned}
$$

We can obtain similar formulas for the other terms on the RHS of (3.9). The result follows from this and inequality (3.9). 
Corollary 3.1. If $\varepsilon>0$ is sufficiently small and $d \geqslant 3$, then the limit

$$
\lim _{n \rightarrow \infty, t=2^{n}} Z_{x, V}(t)=Z_{x, V}(\infty)
$$

exists and $0<Z_{x, V}(\infty)<\infty$ with probability 1 .

Proof. The existence of the limit follows immediately from Proposition 3.3 by standard argument. The fact that $0<Z_{x, y}(\infty)<\infty$ is a consequence of Propositions 3.1 and 3.2.

\section{CONVERGENCE TO DIFFUSION}

In this section we shall prove Theorem 1.1. First we write

$$
\begin{aligned}
& \frac{E_{x}\left[\exp \left[\int_{0}^{N} \varepsilon V(s, X(s)) d s\right] f((X(N)-x) / \sqrt{N})\right]}{E_{x}\left[\exp \left[\int_{0}^{N} \varepsilon V(s, X(s)) d s\right]\right]} \\
& =\frac{E_{x}\left[\exp \left[\int_{0}^{N} \varepsilon V(s, X(s)) d s\right] f((X(N)-x) / \sqrt{N})\right]}{E_{x} E_{V}\left[\exp \left[\int_{0}^{N} \varepsilon V(s, X(s)) d s\right]\right]} / Z_{x, V}(N) \\
& =\frac{Z_{x, V}\left(\left[N^{\prime}\right]\right)}{Z_{x, V}[N]} E_{x}\left[\exp \left[\int_{0}^{N} \varepsilon V(s, X(s)) d s\right] f\left(\frac{X(N)-x}{\sqrt{N}}\right)\right] \\
& \quad \times E_{x} E_{V}\left[\exp \left[\int_{0}^{\left[N^{\prime}\right]} \varepsilon V(s, X(s)) d s\right]\right. \\
& \quad \times\left(E_{x} E_{V}\left[\exp \left[\int_{0}^{N} \varepsilon V(s, X(s)) d s\right] E_{x}\left[\exp \left[\int_{0}^{\left[N^{\prime}\right]} \varepsilon V(s, X(s)) d s\right]\right]\right)^{-1}\right.
\end{aligned}
$$

where $0<\gamma<1$ and $[\cdot]$ denotes integer part. Now let us define

$$
\begin{aligned}
\rho_{M, V}(y)= & \frac{E_{x}\left[\exp \left[\int_{0}^{M} \varepsilon V(s, X(s)) d s\right] ; X(M)=y\right]}{E_{x}\left[\exp \left[\int_{0}^{M} \varepsilon V(s, X(s)) d s\right]\right]} \\
g_{N, \gamma}(y)= & E_{x} E_{V}\left[\exp \left[\int_{0}^{\left[N^{\prime \prime}\right]} \varepsilon V(s, X(s)) d s\right]\right] \\
& \times \frac{E_{y} E_{V}\left[\int_{\left[N^{\prime}\right]}^{N} \varepsilon V(s, X(s)) d s\right]}{E_{x} E_{V}\left[\exp \left[\int_{0}^{N} \varepsilon V(s, X(s)) d s\right]\right]}
\end{aligned}
$$


Then we have that the expression (4.1) is the same as

$$
\begin{aligned}
& \frac{Z_{x, V}\left(\left[N^{\prime}\right]\right)}{Z_{x, V}[N]} \int_{\mathbb{R}^{\prime \prime}} d y \rho_{\left[N^{\prime}\right] . V}(y) g_{N .,}(y) \\
& \quad \times \frac{E_{y}\left[\exp \left[\int_{\left[N^{\prime}\right]}^{N} \varepsilon V(s, X(s)) d s\right] f((X(N)-x) / \sqrt{N})\right]}{E_{V} E_{y}\left[\exp \left[\int_{\left[N^{\prime}\right]}^{N} \varepsilon V(s, X(s)) d s\right]\right]}
\end{aligned}
$$

Now let us define a function $q_{M, v}(y)$ for $y \in \mathbb{R}^{d}$ by

$$
q_{M, V}(y)=\frac{E_{x}\left[\exp \left[\int_{0}^{M} \varepsilon V(s, X(s)) d s\right] ; X(M)=y\right]}{E_{x} E_{V}\left[\exp \left[\int_{0}^{M} \varepsilon V(s, X(s)) d s\right]\right]}
$$

It is evident that

$$
q_{M, V}(y)=Z_{x, V}(M) \rho_{M, V}(y), \quad y \in \mathbb{R}^{d}
$$

observe that

$$
\int_{\mathbb{B}^{d}} p_{M . V}(y) d y=1
$$

and hence we do not expect the integral of $q_{M, V}$ to be unity.

Lemma 4.1. Let $\delta>0$. Then there exist constants $C, c$ depending only on $\delta$ such that

$$
E\left[\int_{|y-x|>M^{12+\delta}} q_{M, v}(y) d y\right] \leqslant C \exp \left[-c M^{\delta}\right]
$$

Proof. We have

$$
E\left[\int_{|y-x|>M^{12+\delta}} q_{M, \nu}(y) d y\right]=\int_{|y-x|>M^{12+\delta}} \rho_{M}(y) d y
$$

where $\rho_{M}$ is as in Proposition 2.2. The result follows immediately now from this proposition.

Lemma 4:2. Let $I_{N, V}$ be the expression

$$
\begin{aligned}
I_{N, V}= & \int_{\mathbb{R}^{d}} d y q_{\left[N^{\prime}\right], V}(y) g_{N, y}(y) \\
& \times\left\{E_{y}\left[\exp \left[\int_{\left[N^{\prime}\right]}^{N} \varepsilon V(s, X(s)) d s\right] f((X(N)-x) / \sqrt{N})\right]\right.
\end{aligned}
$$




$$
\begin{aligned}
& \left.-E_{V} E_{y}\left[\exp \left[\int_{\left[N^{\prime}\right]}^{N} \varepsilon V(s, X(s)) d s\right] f((X(N)-x) / \sqrt{N})\right]\right\} \\
& \times\left(E_{V} E_{y}\left[\exp \left[\int_{\left[N^{\prime \prime}\right]}^{N} \varepsilon V(s, X(s)) d s\right]\right]\right)^{-1}
\end{aligned}
$$

Then there exist positive constants $C, \alpha$ such that

$$
E\left[I_{N, V}^{2}\right] \leqslant C / N^{\alpha}
$$

Proof. We consider first

$$
\begin{aligned}
E\left[I_{N, V}^{2} \mid\right. & \left.V(s, \cdot), s \leqslant\left[N^{\prime}\right]\right] \\
= & \int_{\mathbb{R}^{\prime}} \int_{\mathbb{R}^{d}} d y d z q_{\left[N^{\prime}\right], V}(y) g_{N, \gamma^{\prime}}(y) q_{\left[N^{\prime}\right] . V}(z) g_{N, \gamma^{\prime}}(z) \\
& \times E_{y,=}^{4}\left[\left\{E_{V}\left[\exp \left[\int_{0}^{N-\left[N^{\prime \prime}\right]} \varepsilon V(s, X(s)) d s+\varepsilon V(s, Y(s)) d s\right]\right]\right.\right. \\
& \times\left(E_{V}\left[\exp \left[\int_{0}^{N-\left[N^{\prime}\right]} \varepsilon V(s, X(s)) d s\right]\right]\right. \\
& \left.\left.\times E_{V}\left[\exp \left[\int_{0}^{N-\left[N^{\prime}\right]} \varepsilon V(s, Y(s)) d s\right]\right]\right)^{-1}-1\right\} \\
& \left.\times f\left(\left(X\left(N-\left[N^{\prime}\right]\right)-x\right) / \sqrt{N}\right) f\left(\left(Y\left(N-\left[N^{\prime}\right]\right)-x\right) / \sqrt{N}\right)\right]
\end{aligned}
$$

In the last equation $X(s), Y(s)$ are paths which run for time $s \leqslant N-\left[N^{\prime}\right]$ with $X(0)=y, Y(0)=z$. In view of (3.5) it follows that

$$
\begin{aligned}
& E\left[I_{N . V^{\prime}}^{2} \mid V(s, \cdot), s \leqslant\left[N^{\prime}\right]\right] \\
& \leqslant\left\|g_{N_{.} ;}\right\|_{\infty}^{2} \int_{\mathbb{R}^{\prime}} \int_{\mathbb{R}^{\prime \prime}} d y d z q_{\left[N^{\prime \prime}\right] . V}(y) q_{\left[N^{\prime}\right] . V}(z)
\end{aligned}
$$

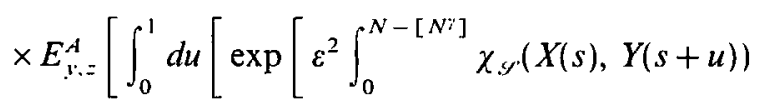

$$
\begin{aligned}
& \left.\left.+\chi_{S,}(X(s+u), Y(s)) d s\right]-1\right] \\
& \left.\times|f|\left(\left(X\left(N-\left[N^{\prime}\right]\right)-x\right) / \sqrt{N}\right)|f|\left(\left(Y\left(N-\left[N^{\prime}\right]\right)-x\right) / \sqrt{N}\right)\right]
\end{aligned}
$$


Hence we have

$$
\begin{aligned}
E\left[I_{N, V}^{2}\right]= & E\left[E\left[I_{N, V}^{2} \mid V(s, \cdot), s \leqslant\left[N^{\alpha}\right]\right]\right] \\
\leqslant & \left\|g_{N, \gamma}\right\|_{\infty}^{2} \int_{\mathbb{R}^{\prime}} \int_{\mathbb{R}^{\prime \prime}} d y d z \rho_{\left[N^{\prime}\right]}(y) \rho_{\left[N^{\prime}\right]}(z) \\
& \times E_{y, z}^{A}\left[\int _ { 0 } ^ { 1 } d u \left[\operatorname { e x p } \left[\varepsilon^{2} \int_{0}^{N-\left[N^{\prime}\right]} \chi_{s,}(X(s), Y(s+u))\right.\right.\right. \\
& \left.\left.+\chi_{s,}(X(s+u), Y(s)) d s\right]-1\right] \\
& \left.\times|f|\left(\left(X\left(N-\left[N^{\prime}\right]\right)-x\right) / \sqrt{N}\right)|f|\left(\left(Y\left(N-\left[N^{\prime}\right]\right)-x\right) / \sqrt{N}\right)\right]
\end{aligned}
$$

Applying the Schwarz inequality to this last expression, we see that

$$
\begin{aligned}
E\left[I_{N, y}^{2}\right] \leqslant & \left\|g_{N, y}\right\|_{\infty}^{2}\left[\int_{\mathbb{Q}^{d}} d y\right. \\
& \left.\times \rho_{\left[N^{\prime}\right]}(y) E_{y^{A}}^{A}\left[|f|^{2}\left(\left(X\left(N-\left[N^{\prime}\right]\right)-x\right) / \sqrt{N}\right)\right]\right] \\
& \times\left\{\int_{0}^{1} d u \int_{\mathbb{R}^{\prime l}} \int_{\mathbb{R}^{d}} d y d z \rho_{\left[N^{\prime}\right]}(y) \rho_{\left[N^{\prime}\right]}(z)\right. \\
& \times E_{y=}^{A}\left[\operatorname { e x p } \left[2 \varepsilon^{2} \int_{0}^{N-\left[N^{\prime \prime}\right]} \chi_{, y}(X(s), Y(s+u))\right.\right. \\
& \left.\left.+\chi_{, y}(X(s+u), Y(s) d s]-1\right]\right\}^{1 / 2}
\end{aligned}
$$

The inequality (4.4) follows from this last inequality by virtue of Proposition 2.2 and Theorem 2.1.

Lemma 4.3. There exist constants $C, \alpha>0$ such that

$$
E\left[\left\{\int_{\mathbb{R}^{d}} d y q_{\left[N^{\prime}\right], \nu}(y)\left[g_{N, y}(y)-1\right]\right\}^{2}\right] \leqslant C / N^{\alpha}
$$

Proof. We consider first

$$
E\left[\int_{\mathbb{R}^{d}} d y q_{\left[N^{\prime}\right], r}(y) g_{N, \gamma}(y)\right]=A_{0}^{\left[N^{\prime}\right]} g_{N, \gamma}(x) / A_{0}^{\left[N^{\prime}\right]} \chi_{0}(x)
$$


It is easy to see that

$$
g_{N ., y}(y)=\left\langle\chi_{0}, u_{0}\right\rangle u_{0}(y)+O\left(e^{-c N}\right)
$$

for some positive $c$. Thus we have

$$
E\left[\int_{\mathbb{R}^{\prime \prime}} d y q_{\left[N^{\prime}\right], V}(y) g_{N ., y}(y)\right]=1+O\left(\exp \left(-c N^{\prime}\right)\right)
$$

Next we use the notation of Proposition 3.3 to write

$$
E\left[\left\{\int_{\mathbb{P}^{d}} d y q_{\left[N^{\prime}\right], V}(y)\right\}^{2}\right]=E_{x, x}^{A,\left[N^{\prime}\right],\left[N^{\prime}\right]}\left[\prod_{i=1}^{\left[N_{i}^{i}\right]} g_{i}(X, Y)\right]
$$

Just as in Proposition 3.3, we have

$$
\begin{aligned}
& E_{x, Y}^{\mathcal{A},\left[N^{\prime}\right],\left[N^{\prime}\right]}\left[\prod_{i=1}^{\left[N^{\prime}\right]} g_{i}(X, Y)\right] \\
& =E_{x ; x^{\prime}}^{A,\left[N^{i}\right] \cdot\left[N^{7}\right]}\left[\prod_{i=1}^{\left[N^{7 / 2}\right]} g_{i}(X, Y)\right]+O\left(1 / N^{x}\right) \\
& \times E_{* i . .}^{A,[N / / 2] .\left[N^{i / 2}\right]}\left[\prod_{i=1}^{[N i / 2]} g_{i}(X, Y)\right]\left\langle\chi_{0}, u_{0}\right\rangle^{2} \\
& \times u_{0}\left(X\left(\left[N^{\prime} / 2\right]\right)\right) u_{0}\left(Y\left(\left[N^{\prime} / 2\right]\right)+O\left(1 / N^{\alpha}\right)=a_{N}+O\left(1 / N^{\alpha}\right)\right.
\end{aligned}
$$

where $a_{N}$ denotes the expectation value in the last expression. Similarly we have

$$
\begin{array}{r}
E\left[\left\{\int_{\mathbb{R}^{d}} d y q_{\left[N^{\prime}\right], v}(y) g_{N, \gamma}(y)\right\}^{2}\right]=a_{N}+O\left(1 / N^{\alpha}\right) \\
E\left[\int_{\mathbb{R}^{d}} d y q_{\left[N^{\prime}\right], V}(z) \int_{\mathbb{R}^{d}} d z q_{\left[N^{y}\right], \nu}(z) g_{N, y}(z)\right]=a_{N}+O\left(1 / N^{\alpha}\right)
\end{array}
$$

The result follows now from the last three equations.

Proof of Theorem 1.1. We start from the expression (4.2). First observe that by Corollary 3.1 we have

$$
\lim _{n \rightarrow \infty, N=2^{n}} Z_{x, y}\left(\left[N^{\prime}\right]\right) / Z_{x, V}(N)=1
$$


with probability 1 . Now let us put

$$
\begin{aligned}
a_{N}= & \int_{\mathbb{R}^{d}} d y \rho_{\left[N^{\prime}\right], V}(y) g_{N, y}(y) \\
& \times \frac{E_{y}\left[\exp \left[\int_{\left[N^{\prime}\right]}^{N} \varepsilon V(s, X(s)) d s\right] f((X(N)-x) / \sqrt{N})\right]}{E_{V} E_{y}\left[\exp \left[\int_{\left[N^{\prime}\right]}^{N} \varepsilon V(s, X(s)) d s\right]\right]} \\
b_{N}= & \int_{\mathbb{R}^{d}} d y \rho_{\left[N^{\prime}\right], V}(y) g_{N, \gamma}(y) \\
& \times \frac{E_{V} E_{y}\left[\exp \left[\int_{\left[N^{\prime}\right]}^{N} \varepsilon V(s, X(s)) d s\right] f((X(N)-x) / \sqrt{N})\right]}{E_{V} E_{y}\left[\exp \left[\int_{\left[N^{\prime}\right]}^{N} \varepsilon V(s, X(s)) d s\right]\right]}
\end{aligned}
$$

We need to show that $\lim _{n \rightarrow \infty, N=2^{n}} a_{N}$ exists and is equal to the RHS of (1.12), which we denote by $\mathscr{L}$. Now by Proposition $3.2,(4.2)$, and Lemma 4.2 it is sufficient to show that $\lim _{n \rightarrow \infty, N=2^{n}} b_{N}$ exists and is equal to $\mathscr{L}$. Next we define $c_{N}$ by

$$
\begin{aligned}
c_{N}= & \int_{|x-y|<N^{y / 2+\delta}} d y \rho_{\left[N^{\prime}\right], V}(y) g_{N, y^{\prime}}(y) \\
& \times E_{V} E_{y}\left[\exp \left[\int_{\left[N^{i}\right]}^{N} \varepsilon V(s, X(s)) d s\right]\right] \\
& \times f(X(N)-x) / \sqrt{N}) / E_{V} E_{y}\left[\exp \left[\int_{\left[N^{\prime}\right]}^{N} \varepsilon V(s, X(s)) d s\right]\right]
\end{aligned}
$$

where $\delta>0$. Then by Lemma 4.1, (4.3), and Proposition 3.2, it is sufficient to show that $\lim _{n \rightarrow \infty, N=2^{n}} c_{N}$ exists and is equal to $\mathscr{L}$. Observe next that if $\gamma / 2+\delta<1 / 2$, then

$$
\begin{aligned}
& \sup _{\left|x-y^{\prime}\right|<N^{\gamma / 2+\delta}} \mid E_{V} E_{y^{\prime}}\left[\exp \left[\int_{\left[N^{\prime}\right]}^{N} \varepsilon V(s, X(s)) d s\right]\right] \\
& \quad \times f(X(N)-x) / \sqrt{N}) / E_{V} E_{y}\left[\exp \left[\int_{\left[N^{\prime}\right]}^{N} \varepsilon V(s, X(s)) d s\right]\right]-\mathscr{L} \mid
\end{aligned}
$$

converges to zero as $N \rightarrow \infty$. Thus if we put

$$
d_{N}=\int_{|x-y|<N^{2 / 2+s}} d y \rho_{\left[N^{i}\right], \nu}(y) g_{N, y}(y)
$$


it is sufficient to prove $\lim _{n \rightarrow \infty . N=2^{n}} d_{N}=1$. Again by Lemma 4.1 this is the same as showing that the sequence

$$
e_{N}=\int_{\mathbb{R}^{d}} d y \rho_{\left[N^{\prime}\right], v}(y) g_{N .,}(y)
$$

converges to 1 as $N$ goes exponentially fast to $\infty$. However, this last fact is a consequence of Lemma 4.3. QED

\section{APPENDIX. EXISTENCE OF SPECTRAL GAP}

Here we shall show that the quantity $\lambda_{2}$ defined by

$$
\lambda_{2}=\left\langle u_{0}, A_{2} u_{0}\right\rangle+\left\langle A_{1} u_{0},\left(\left\|A_{0}\right\|-A_{0}\right)^{-1} A_{1} u_{0}\right\rangle
$$

is strictly negative.

Our first goal will be to prove that $\lambda_{2} \leqslant 0$. To do this let $\xi=t n$, where $t \in \mathbb{R}$ and $n \in \mathbb{R}^{d}$ is a unit vector. Suppose $v \in L^{2}\left(Q_{0}\right)$ is a function which is pure imaginary and orthogonal to $u_{0}$. It is clear that

$$
\begin{aligned}
\left\langle u_{0}+t v, A_{\xi}\left(u_{0}+t v\right)\right\rangle & \leqslant\left\langle\left|u_{0}+t v\right|, A_{0}\left|u_{0}+t v\right|\right\rangle \\
& \leqslant\left\|A_{0}\right\| \cdot\left\|u_{0}+t v\right\|^{2} \\
& =\left\|A_{0}\right\|\left[\left\|u_{0}\right\|^{2}+t^{2}\|v\|^{2}\right]
\end{aligned}
$$

where $\left|u_{0}+t v\right|$ is the function defined by

$$
\left|u_{0}+t v\right|(x)=\left|u_{0}(x)+t v(x)\right|, \quad x \in \mathbb{R}^{d}
$$

We have now that

$$
\begin{aligned}
\left\langle u_{0}\right. & \left.+t v, A_{\xi}\left(u_{0}+t v\right)\right\rangle \\
& =\left\langle u_{0}+t v,\left(A_{0}+t A_{1}+t^{2} A_{2}\right)\left(u_{0}+t v\right)\right\rangle+O\left(t^{3}\right) \\
& =\left\|A_{0}\right\| \cdot\left\|u_{0}\right\|^{2}+t^{2}\left\langle v, A_{0} v\right\rangle+2 t^{2}\left\langle u_{0}, A_{1} v\right\rangle+t^{2}\left\langle u_{0}, A_{2} u_{0}\right\rangle+O\left(t^{3}\right)
\end{aligned}
$$

In this last identity we have used the fact that $v$ is pure imaginary, since it implies that $\left\langle u_{0}, A_{1} v\right\rangle$ is a real number. Now if we let $t \rightarrow 0$ in (A.1) and (A.2) we easily see that

$$
\left\langle u_{0}, A_{2} u_{0}\right\rangle+2\left\langle u_{0}, A_{1} v\right\rangle-\left\langle v,\left(\left\|A_{0}\right\|-A_{0}\right) v\right\rangle \leqslant 0
$$

Taking $v=\left(\left\|A_{0}\right\|-A_{0}\right)^{-1} A_{1} u_{0}$ in this last inequality yields $\lambda_{2} \leqslant 0$. 
In order to prove that $\lambda_{2}<0$, we need to obtain a formula for the difference between the LHS and RHS of (A.1). We have that

$$
\left\langle u_{0}+t v, A_{\xi}\left(u_{0}+t v\right)\right\rangle=\int_{Q_{0}} \int_{Q_{0}} d x d y \sum_{m \in \mathbb{Z}^{d}} f(x, y, m)
$$

where

$$
f(x, y, m)=\left[u_{0}(x)-t v(x)\right] K(x, y+m) e^{i \xi \cdot m}\left[u_{0}(y)+t v(y)\right]
$$

Hence we have

$$
\begin{aligned}
\left.K u_{0}+t v, A_{\xi}\left(u_{0}+t v\right)\right\rangle\left.\right|^{2} \\
=\left|\int_{Q_{0}} \int_{Q_{0}} d x d y \sum_{m \in \mathbb{Z}^{d}} f(x, y, m)\right|^{2} \\
=\left[\int_{Q_{0}} \int_{Q_{0}} d x d y \sum_{m \in \mathbb{Z}^{\prime}}|f(x, y, m)|^{2}\right. \\
\quad-\frac{1}{2} \int_{Q_{0}} \int_{Q_{0}} d x d y \sum_{m \in \mathbb{Z}^{d}} \int_{Q_{0}} \int_{Q_{0}} d x^{\prime} d y^{\prime} \\
\quad \times \sum_{m^{\prime} \in \mathbb{Z}^{d}} \frac{|| f(x, y, m)\left|f\left(x^{\prime}, y^{\prime}, m^{\prime}\right)-\right| f\left(x^{\prime}, y^{\prime}, m^{\prime}\right)|f(x, y, m)|^{2}}{|f(x, y, m)| \cdot\left|f\left(x^{\prime}, y^{\prime}, m^{\prime}\right)\right|}
\end{aligned}
$$

It is clear that

$$
|f(x, y, m)|=\left[u_{0}(x)^{2}+t^{2}|v(x)|^{2}\right]^{1 / 2} K(x, y+m)\left[u_{0}(y)^{2}+t^{2}|v(y)|^{2}\right]^{1 / 2}
$$

Consider the function $g_{1}(x)$ defined by

$$
g_{l}(x)=\left[u_{0}(x)^{2}+t^{2}|v(x)|^{2}\right]^{1 / 2}-u_{0}(x)
$$

Then we have

$$
\begin{array}{rl}
\int_{Q_{0}} \int_{Q_{0}} & d x \dot{d} y \sum_{m \in \mathbb{Z}^{d}}|f(x, y, m)| \\
& =\left\langle g_{t}+u_{0}, A_{0}\left(g_{t}+u_{0}\right)\right\rangle \\
& =\left\|A_{0}\right\|\left[\left\|u_{0}\right\|^{2}+t^{2}\|v\|^{2}\right]+\left\langle g_{t}+u_{0},\left(A_{0}-\left\|A_{0}\right\|\right)\left(g_{t}+u_{0}\right)\right\rangle \\
& =\left\|A_{0}\right\|\left[\left\|u_{0}\right\|^{2}+t^{2}\|v\|^{2}\right]+\left\langle g_{t},\left(A_{0}-\left\|A_{0}\right\|\right) g_{t}\right\rangle
\end{array}
$$


Since $g_{t}=O\left(t^{2}\right)$, we conclude that

$$
\int_{Q_{0}} \int_{Q_{0}} d x d y \sum_{m \in \mathbb{Z}^{d}}|f(x, y, m)|=\left\|A_{0}\right\|\left[\left\|u_{0}\right\|^{2}+t^{2}\|v\|^{2}\right]+O\left(t^{4}\right)
$$

Next we write

$$
f(x, y, m)=a_{0}+t a_{1}+O\left(t^{2}\right)
$$

Since

$$
\begin{aligned}
f(x, y, m)= & {\left[u_{0}(x)-t v(x)\right] K(x, y+m) } \\
& \times[1+i t m \cdot n]\left[u_{0}(y)+t v(y)\right]+O\left(t^{2}\right)
\end{aligned}
$$

it follows that

$$
\begin{aligned}
& a_{0}=u_{0}(x) K(x, y+m) u_{0}(y) \\
& a_{1}=i(m \cdot n) a_{0}+u_{0}(x) K(x, y+m) v(y)-v(x) K(x, y+m) u_{0}(y)
\end{aligned}
$$

Observe that $a_{0}$ is real while $a_{1}$ is pure imaginary. We conclude that

$$
\begin{aligned}
|f(x, y, m)| f\left(x^{\prime}, y^{\prime}, m^{\prime}\right)-\left|f\left(x^{\prime}, y^{\prime}, m^{\prime}\right)\right| f(x, y, m) \\
\quad=a_{0}\left[a_{0}^{\prime}+t a_{1}^{\prime}\right]-a_{0}^{\prime}\left[a_{0}+t a_{1}\right]+O\left(t^{2}\right) \\
\quad=t\left[a_{0} a_{1}^{\prime}-a_{1}\right]+O\left(t^{2}\right)
\end{aligned}
$$

where $a_{0}^{\prime}, a_{1}^{\prime}$ are the quantities for $f\left(x^{\prime}, y^{\prime}, m^{\prime}\right)$ corresponding to $a_{0}, a_{1}$. Hence

$$
\begin{aligned}
& \frac{|| f(x, y, m)\left|f\left(x^{\prime}, y^{\prime}, m^{\prime}\right)-\right| f\left(x^{\prime}, y^{\prime}, m^{\prime}\right)|f(x, y, m)|^{2}}{|f(x, y, m)| \cdot\left|f\left(x^{\prime}, y^{\prime}, m^{\prime}\right)\right|} \\
& =t^{2} \frac{\left|a_{0} a_{1}^{\prime}-a_{0}^{\prime} a_{1}\right|^{2}}{a_{0} a_{0}^{\prime}}+O\left(t^{3}\right)
\end{aligned}
$$

It follows now from (A.3)-(A.5) and the previous equation that

$$
\begin{aligned}
\left|\left\langle u_{0}+t v, A_{\xi}\left(u_{0}+t v\right)\right\rangle\right|^{2} & =\left(\left\|A_{0}\right\|\left[\left\|u_{0}\right\|^{2}+t^{2}\|v\|^{2}\right]\right)^{2} \\
& \quad-\frac{t^{2}}{2} \int_{Q_{0}} \int_{Q_{0}} d x d y \sum_{m \in \mathbb{Z}^{d}} \int_{Q_{0}} \int_{Q_{0}} d x^{\prime} d y^{\prime}
\end{aligned}
$$




$$
\begin{aligned}
& \times \sum_{m^{\prime} \in \mathbb{Z}^{d}} K(x, y+m) K\left(x^{\prime}, y^{\prime}+m^{\prime}\right) \\
& \times \mid u_{0}(x) u_{0}(y) u_{0}\left(x^{\prime}\right) u_{0}\left(y^{\prime}\right) i\left(m^{\prime} \cdot n-m \cdot n\right) \\
& +u_{0}(x) u_{0}(y) u_{0}\left(x^{\prime}\right) v\left(y^{\prime}\right)-u_{0}\left(x^{\prime}\right) u_{0}\left(y^{\prime}\right) u_{0}(x) v(y) \\
& -u_{0}(x) u_{0}(y) v\left(x^{\prime}\right) u_{0}\left(y^{\prime}\right)+\left.u_{0}\left(x^{\prime}\right) u_{0}\left(y^{\prime}\right) v(x) u_{0}(y)\right|^{2} \\
& \times\left[u_{0}(x) u_{0}(y) u_{0}\left(x^{\prime}\right) u_{0}\left(y^{\prime}\right)\right]^{-1}+O\left(t^{3}\right)
\end{aligned}
$$

From (A.2) we have

$$
\begin{aligned}
K\left\langle u_{0}+\right. & \left.t v, A_{\xi}\left(u_{0}+t v\right)\right\rangle\left.\right|^{2} \\
= & \left(\left\|A_{0}\right\| \cdot\left\|u_{0}\right\|^{2}\right)^{2} \\
& +2\left\|A_{0}\right\| \cdot\left\|u_{0}\right\|^{2} t^{2}\left[\left\langle v, A_{0} v\right\rangle+2\left\langle u_{0}, A_{1} v\right\rangle+\left\langle u_{0}, A_{2} u_{0}\right\rangle\right]+O\left(t^{3}\right)
\end{aligned}
$$

Comparing the coefficients of $t^{2}$ in the last two identities, we see that

$$
\begin{array}{r}
\left\langle v, A_{0} v\right\rangle+2\left\langle u_{0}, A_{1} v\right\rangle+\left\langle u_{0}, A_{2} u_{0}\right\rangle \\
=\left\|A_{0}\right\| \cdot\|v\|^{2}-\frac{1}{4\left\|A_{0}\right\| \cdot\left\|u_{0}\right\|^{2}} I(v)
\end{array}
$$

where

$$
\begin{aligned}
I(v)= & \int_{Q_{0}} \int_{Q_{0}} d x d y \sum_{m \in \mathbb{Z}^{d}} \int_{Q_{0}} \int_{Q_{0}} d x^{\prime} d y^{\prime} \\
& \times \sum_{m^{\prime} \in \mathbb{Z}^{d}} K(x, y+m) K\left(x^{\prime}, y^{\prime}+m^{\prime}\right) \\
& \times \mid u_{0}(x) u_{0}(y) u_{0}\left(x^{\prime}\right) u_{0}\left(y^{\prime}\right) i\left(m^{\prime} \cdot n-m \cdot n\right) \\
& +u_{0}(x) u_{0}(y) u_{0}\left(x^{\prime}\right) v\left(y^{\prime}\right)-u_{0}\left(x^{\prime}\right) u_{0}\left(y^{\prime}\right) u_{0}(x) v(y) \\
& -u_{0}(x) u_{0}(y) v\left(x^{\prime}\right) u_{0}\left(y^{\prime}\right)+\left.u_{0}\left(x^{\prime}\right) u_{0}\left(y^{\prime}\right) v(x) u_{0}(y)\right|^{2} \\
& \times\left[u_{0}(x) u_{0}(y) u_{0}\left(x^{\prime}\right) u_{0}\left(y^{\prime}\right)\right]^{-1}
\end{aligned}
$$

We shall show that $I(v)>0$ if $v \neq 0$. To see this, we bound $I(v)$ below by the sum restricted to $m=m^{\prime}$. Thus, if

$$
\begin{aligned}
h\left(x, y, x^{\prime}, y^{\prime}\right)= & u_{0}(x) u_{0}(y) u_{0}\left(x^{\prime}\right) v\left(y^{\prime}\right) \\
& -u_{0}\left(x^{\prime}\right) u_{0}\left(y^{\prime}\right) u_{0}(x) v(y)-u_{0}(x) u_{0}(y) v\left(x^{\prime}\right) u_{0}\left(y^{\prime}\right) \\
& +u_{0}\left(x^{\prime}\right) u_{0}\left(y^{\prime}\right) v(x) u_{0}(y)
\end{aligned}
$$


we have

$$
\begin{aligned}
I(v) \geqslant & \int_{Q_{0}} \int_{Q_{0}} d x d y \int_{Q_{0}} \int_{Q_{0}} d x^{\prime} d y^{\prime} \\
& \times \sum_{m \in \mathbb{Z}^{d}} K(x, y+m) K\left(x^{\prime}, y^{\prime}+m\right)\left|h\left(x, y, x^{\prime}, y^{\prime}\right)\right|^{2} \\
& \times\left[u_{0}(x) u_{0}(y) u_{0}\left(x^{\prime}\right) u_{0}\left(y^{\prime}\right)\right]^{-1}
\end{aligned}
$$

Observe next that

$$
\begin{aligned}
& h\left(x, y, x^{\prime}, y\right) \\
& \quad=-u_{0}(x) u_{0}(y) v\left(x^{\prime}\right) u_{0}(y)+u_{0}\left(x^{\prime}\right) u_{0}(y) v(x) u_{0}(y) \\
& \quad=u_{0}(y)^{2}\left[-u_{0}(x) v\left(x^{\prime}\right)+u_{0}\left(x^{\prime}\right) v(x)\right]
\end{aligned}
$$

If $I(v)=0$, then we must have

$$
u_{0}(x) v\left(x^{\prime}\right)=u_{0}\left(x^{\prime}\right) v(x), \quad x, x^{\prime} \in Q_{0}
$$

whence $v(x)=c u_{0}(x)$ for some constant $c$. It is easy to see that $I\left(u_{0}\right)>0$. Hence $I(v)>0$ all $v \neq 0$.

We finally conclude that $\lambda_{2}<0$ since

$$
v=\left(\left\|A_{0}\right\|-A_{0}\right)^{-1} A_{1} u_{0} \neq 0
$$

\section{ACKNOWLEDGMENTS}

The authors would like to thank Renming Song for pointing out an error in a previous version of the paper. The research of J.G.C. was partially supported by U.S. National Science Foundation grant DMS9100455. The research of P.A.O. was supported by NFR-Norges Forskningsåd (Norwegian Research Council) grant 100222/410.

\section{REFERENCES}

1. E. Bolthausen, A note on the diffusion of directed polymers in a random environment, Commun. Math. Phys. 123:529-534 (1989).

2. L. Breiman, Probability (Addison-Wesley, Reading, Massachusetts, 1968).

3. J. Z. Imbrie and T. Spencer, Diffusion of directed polymers in a random environment, J. Stat. Phys. 52:609-626 (1988).

4. M. Krasnoselskii, Positive Solutions of Operator Equations (Noordhoff, Groningen, 1964).

5. D. Ruelle, Statistical Mechanics-Rigorous Results (Benjamin, 1969).

6. A. Taylor, Functional Analysis (Wiley, New York, 1958). 